\title{
Le haut lieu touristique comme objet spatial linéaire : le somin Volcan (île de La Réunion)
}

Fabrication, banalisation et patrimonialisation

From acclaimed tourist destination to linear spatial construct: The Somin Volcano (Reunion Island)

Construction, banalization and heritagization

El sitio turístico privilegiado como objeto espacial lineal: El Somin Volcan (Isla de La Reunión) Fabricación, banalizaciôn y patrimonialización

\section{Christian Germanaz}

Volume 57, numéro 162, décembre 2013

Version originale soumise en juin 2013.

Version révisée reçue en janvier 2014.

URI : https://id.erudit.org/iderudit/1026525ar

DOI : https://doi.org/10.7202/1026525ar

Aller au sommaire du numéro

Éditeur(s)

Département de géographie de l’Université Laval

ISSN

0007-9766 (imprimé)

1708-8968 (numérique)

Découvrir la revue

Citer cet article

Germanaz, C. (2013). Le haut lieu touristique comme objet spatial linéaire : le somin Volcan (île de La Réunion) : fabrication, banalisation et patrimonialisation. Cahiers de géographie du Québec, 57(162), 379-405. https://doi.org/10.7202/1026525ar

\section{Résumé de l'article}

La route, qu'elle soit du sel ou de la soie, la ligne, céleste de Mermoz ou océanique des Polynésiens, le chemin, qu'il mène à Compostelle ou à Machu Picchu, tous participent depuis très longtemps au développement d'un imaginaire collectif attaché au voyage et à l'ailleurs. Le regain d'intérêt pour les routes touristiques et les itinéraires culturels résulte de l'attention particulière que leur accordent les voyagistes de la planète tourisme, obsédés par le renouvellement permanent de leur offre de destinations. À travers l'exemple du somin Volcan (île de La Réunion), nous nous interrogeons sur les processus à l'origine des pratiques et des représentations spatiales conduisant une structure géographique élémentaire comme la ligne à devenir un itinéraire identitaire aux caractères touristiques affirmés. La morphologie et les dynamiques de cet objet spatial, ancré à la fois dans le passé et le présent, sont interpellées pour aider à comprendre la transformation des spatialités attachées à l'évolution des pratiques touristiques du somin Volcan. Positionnées entre mémoire et développement et guidant le déroulement de notre analyse, trois périodes ont été identifiées dans cette évolution. La première correspond à la fabrication de l'itinéraire ; elle explique sa pérennité et ses propriétés. La banalisation constitue le second temps, marqué par des postures sociales d'appropriation culturelle de la part des habitants de l'île. Sous la pression contemporaine des politiques de protection environnementale et de patrimonialisation, la dernière époque caractérise la reconfiguration de la nature mythologique du somin Volcan, offrant de nouvelles perspectives aux visiteurs de la Fournaise. Cet exemple, peut-il avoir une valeur heuristique pour appréhender l'essence et les métamorphoses du haut lieu touristique linéarisé ?
Ce document est protégé par la loi sur le droit d'auteur. L'utilisation des services d'Érudit (y compris la reproduction) est assujettie à sa politique d'utilisation que vous pouvez consulter en ligne.

https://apropos.erudit.org/fr/usagers/politique-dutilisation/ 


\title{
Le haut lieu touristique comme objet spatial linéaire: le somin Volcan (île de La Réunion) Fabrication, banalisation et patrimonialisation
}

\author{
From acclaimed tourist destination to \\ linear spatial construct: The Somin Volcano \\ (Reunion Island) \\ Construction, banalization and \\ heritagization \\ El sitio turístico privilegiado como objeto \\ espacial lineal: El Somin Volcan (Isla de La \\ Reunión) \\ Fabricación, banalizaciôn y \\ patrimonialización
}

\author{
Christian GERMANAZ \\ Université de La Réunion \\ Germanaz@univ-reunion.fr
}

\section{Résumé}

La route, qu'elle soit du sel ou de la soie, la ligne, céleste de Mermoz ou océanique des Polynésiens, le chemin, qu'il mène à Compostelle ou à Machu Picchu, tous participent depuis très longtemps au développement d'un imaginaire collectif attaché au voyage et à l'ailleurs. Le regain d'intérêt pour les routes touristiques et les itinéraires culturels résulte de l'attention particulière que leur accordent les voyagistes de la planète tourisme, obsédés par le renouvellement permanent de leur offre de destinations. À travers l'exemple du somin Volcan (île de La Réunion), nous nous interrogeons sur les processus à l'origine des pratiques et des représentations spatiales conduisant une structure géographique élémentaire comme la ligne à devenir un itinéraire identitaire aux caractères touristiques affirmés. La morphologie et les dynamiques de cet objet spatial, ancré à la fois dans le passé et le présent, sont interpellées pour aider à comprendre la transformation des spatialités attachées à l'évolution des pratiques touristiques du somin Volcan. Positionnées entre mémoire et développement et guidant le déroulement de notre analyse, trois périodes ont été identifiées dans cette évolution. La première correspond à la fabrication de l'itinéraire ; elle explique sa pérennité et ses propriétés. La banalisation constitue le second temps, marqué par des postures sociales d'appropriation culturelle de la part des habitants de l'île. Sous la pression contemporaine des politiques de protection environnementale et de patrimonialisation, la dernière époque caractérise la reconfiguration de la nature mythologique du somin Volcan, offrant de nouvelles perspectives aux visiteurs de la Fournaise. Cet exemple, peut-il avoir une valeur heuristique pour appréhender l'essence et les métamorphoses du haut lieu touristique linéarisé?

\section{Mots-clés}

Voyage au volcan, interprétation, mise en scène touristique, patrimoine mondial, Piton de la Fournaise, île de La Réunion. 


\begin{abstract}
The ancient Silk and Salt Routes, the Saint James Way of Compostela, the Trail to Machu Picchu or the sea and air routes traced by the Polynesians and by pioneer aviator Jean Mermoz have been a fundamental part of our collective image of voyaging since time immemorial. Interest in tourist trails or cultural itineraries has risen today as a result of the intense focus put on this type of route by global tourism operators, driven by their need to constantly expand the destination choices they offer. Taking Reunion Island's Somin Volcan as an example, our article examines the spatial practices and representations which initially transform a basic linear geographical structure into an itinerary stamped with its own specific identity and tourist features. The present article draws on the morphology and dynamics of this spatial construct, straddling both the past and the present, to explore the transformation of spatiality linked to the evolution of tourist practices around the Somin Volcan. The transformation process involves three distinct phases that lie between memory and development and will be presented in this article. The first phase involves the construction of the itinerary, based on its sustainability and its properties. The second phase is characterized by the banalization of that itinerary, marked by social postures of cultural appropriation adopted by the inhabitants of the island. In the final phase, the mythological or heritage nature of the Somin Volcan is reconstructed, offering new perspectives to those who visit La Fournaise. Could it be worthwhile to consider using the Somin Volcan as a heuristic example through which we can gain a better understanding of the intrinsic nature of a major linear tourist attraction, and the changes it is subjected to?
\end{abstract}

\title{
Keywords
}

Journeys to volcanos, geographical interpretation, enactment of tourism, world heritage sites, Piton de La Fournaise, Reunion Island.

\section{Resumen}

Desde hace largo tiempo, la ruta de sal o de seda, la línea celeste de Mermoz o aquella oceánica de los polinesios, el camino a Compostela o al Machu Pijchu participan en el desarrollo del imaginario colectivo vinculado al viaje y al más allá. La recrudescencia de interés por las rutas turísticas y los itinerarios culturales resulta de la atención particular de viajeros del planeta turismo, obsesionados por el renuevo permanente de destinaciones. Tomando como ejemplo el Somin Volcan (Isla de la Reunión), el autor se pregunta cuales son los procesos que están al origen de prácticas y representaciones espaciales que conducen una estructura geográfica elemental a un itinerario identitario, con características turísticas definidas. La morfología y los dinamismos pasados y presentes de este objeto espacial, ayudan a comprender las transformaciones espaciales debidas a la evolución de las prácticas turísticas del Somin Volcan. Tres períodos han sido así identificados. Primeramente la fabricación del itinerario, explicando su perennidad y sus propiedades. Luego la banalización marcada por posturas sociales de apropiación cultural por parte de los isleños. Finalmente, bajo la presión contemporánea de políticas de protección del medio y del patrimonio, la reconfiguración mitológica del volcán, lo que ofrece nuevas perspectivas a los visitantes de la Fournaise ¿Puede este ejemplo tener valor heurístico para aprehender la esencia y la metamorfosis de un alto sitio turístico linerizado?

\section{Palabras claves}

Viaje al volcán, interpretación, puesta en escena turística, patrimonio mundial, Piton de la Fournaise, Isla de la reunión. 


\section{Introduction}

L'excursion au Piton de la Fournaise occupe une place privilégiée sinon incontournable dans les pratiques touristiques des visiteurs de l'île de La Réunion. L'attractivité jamais démentie de ce volcan actif relève en grande partie de la fréquence de ses épisodes éruptifs et de son accessibilité sécurisée comme fenêtre spectaculaire sur «l'antre du diable» (Krafft et Krafft, 1981). Lieu tout à la fois de «l'indicible effroi» et du sublime, le Piton de la Fournaise est investi d'une valeur identitaire très forte dans les perceptions de la population réunionnaise. En cristallisant les valeurs de la créolité et en sublimant les fantasmes de la société coloniale (Gamaleya, 1983), au rythme du peuplement de l'île et de sa mise en valeur, il s'est imposé comme l'un des principaux géosymboles de l'espace réunionnais. Sa présence imposante dans le panthéon patrimonial de l'île, dans le cœur des habitants et dans la curiosité des visiteurs n’a été possible que par l'existence d'itinéraires permettant la rencontre, l'exploration et l'appropriation du massif volcanique. Le somin Volcan (chemin du volcan) tient ainsi une place déterminante dans le dispositif touristique de La Réunion. Son intérêt ne résulte pas seulement de sa désignation comme bien du parc national et de son appartenance officielle au patrimoine mondial, mais plus sûrement de sa capacité à mobiliser l'imaginaire affectif lié à la mémoire des «voyages au volcan» et de sa fonction comme révélateur sensible du paysage de la Fournaise.

Dans le cadre du dossier thématique Routes touristiques: lire le passé, lier l'avenir, nous souhaitons contribuer, par ce texte, à une meilleure connaissance de cet objet spatial original qu'est la ligne touristique (itinéraire, route, circuit, chemin). Quelles sont les pratiques et les postures sociales qui l'inventent et la transforment dans le temps? Quelles sont les spatialités (Lussault, 2003) qui lui sont attachées et en quoi contribuent-elles à la géographie? En soutenant la valeur heuristique du somin Volcan, l'analyse peut nous permettre de répondre à ce questionnement et de tester sa pertinence comme modèle d'évolution de l'itinéraire culturel. Dans cet objectif, notre démarche commence par une étude de l'environnement géographique et social des «voyages à la Fournaise» à l'origine du somin Volcan. Les trois périodes qui marquent son évolution jusqu'à nos jours sont développées dans un second point. Elles s'articulent assez bien entre mémoire et développement. La mémoire est sédimentée au cours du premier temps où se réalise la fabrication de l'itinéraire volcanique. La banalisation du somin Volcan correspond au moment de l'ouverture d'une route forestière (1965) inaugurant la seconde période de l'itinéraire, caractérisée par une massification des flux de curieux et entraînant l'Office national des forêts (ONF) à produire un aménagement touristique au parti pris pédagogique assumé. Né du risque des déprédations potentielles induites par la surfréquentation de la route, le troisième temps témoigne de la volonté des responsables politiques et des aménageurs de projeter, pour le somin Volcan, une stratégie de conservation et de patrimonialisation. Accompagnant les trois étapes de cette évolution, les spatialités imprimées par les différents groupes de visiteurs qui se sont succédé au fil du temps sont étudiées dans la discussion conclusive. 


\section{La petite fabrique du somin Volcan}

L'évolution du somin Volcan présentée dans ce développement résulte, pour cette première période, de l'analyse de 129 récits de voyage au volcan et de l'étude d'un corpus iconographique très diversifié (cartes, gravures, dessins, aquarelles et photographies), produits entre 1751 et $1965^{1}$.

La genèse du somin Volcan n'a rien d'exceptionnelle: elle procède de la même impulsion qui anime la mise en place des pistes immémoriales tracées par les premiers hommes pour élargir leurs horizons. En ce sens, la trace ne constitue pas en elle-même la finalité du voyage, elle incarne avant tout un vecteur pour transgresser les limites initiales de notre habitation du monde. Son inscription spatiale durable dans le temps est due principalement à la reconnaissance de l'efficacité de sa fonction originelle de jonction entre les lieux. Ce n'est qu'aujourd'hui, à la faveur des stratégies de patrimonialisation à usage culturel ou écotouristique, que la route, l'itinéraire, le chemin, le circuit ou la ligne sont devenus des destinations convoitées par les touristes séduits par cette forme de nomadisme. Pour La Réunion, la fabrique du somin Volcan prend naissance vers la première moitié du XVIII e siècle dans la continuité du peuplement de l'île en direction du sud. La nécessité ressentie par certains administrateurs de la Compagnie des Indes d'élargir leur connaissance de l'espace qu'ils sont censés mettre en valeur et la garantie de protection qu'ils sont supposés assurer aux nouveaux colons, les poussent à organiser de véritables expéditions pour constater, in situ, les risques potentiels du volcan. Si le premier voyage des officiels date de 1768 , quelques habitants de l'île n'ont pas attendu ces préoccupations de façade pour précéder le gouverneur et son ordonnateur sur la Fournaise $^{2}$. Dans tous les cas, l'organisation pragmatique mais codifiée des voyages au volcan et la morphologie de leur architecture sociale apparaissent comme des clés essentielles pour appréhender la fabrication du somin Volcan, son installation dans la pérennité et l'essence de sa mythification.

\section{La nature géographique du voyage au volcan}

L'inscription progressive du somin Volcan sur le massif du Piton de la Fournaise et sa sédimentation comme itinéraire légendaire se réalisent au cours de quelque 125 expéditions, dispersées sur un peu plus de deux siècles et demi (1751-1965) (Germanaz, 2005a). Employé dès l'origine et jusque dans la première moitié du XXe siècle, le terme "voyage», attribué à une excursion dont le trajet aller-retour ne dépasse pas la trentaine de kilomètres, peut aujourd'hui surprendre. Mais pour les premiers curieux, la visite du volcan nécessite l'organisation d'une véritable

1 On trouvera une présentation détaillée de chacun de ces récits dans l'annexe 1 de notre travail doctoral (2005a). Pour la période 1751-1965, nous avons recensé et analysé 520 images relatives au Piton de la Fournaise: 31 dessins, 66 gravures, 15 aquarelles, 12 lithographies, 291 photographies en noir et blanc, 11 en couleur et 94 documents cartographiques (Ibid.). Pour la présentation de ce corpus iconographique et des méthodologies, on peut consulter notre article publié dans l'ouvrage coordonné par Bertrand et al. (Germanaz, 2009).

2 Le premier voyage au volcan avec une ascension réussie du Piton de la Fournaise a eu lieu en septembre 1751 à l'initiative du Chevalier de Palmeroux, "Gentilhomme Bourguignon, établi dans l'Isle de Bourbon» d'après le mémoire de M. D’Heguerty (1755), ancien commandant de cette île pour le roi. 
expédition projetée sur plusieurs jours, voire sur plus d'une semaine, en prenant comme position de départ, Saint-Denis, le chef-lieu de la petite colonie. Aventure en terra incognita, le projet est aussi élaboré dans un bain de rumeurs qui exacerbe les dangers encourus par les intrépides (ou inconscients) voyageurs (Bory de SaintVincent, 1804: 181), ce qui ajoute à l'entreprise une tension qui apparaît souvent aux yeux des contemporains comme totalement déraisonnable (Hubert, 1799).

En effet, les premiers visiteurs doivent se hasarder sur un terrain encore inexploré dont la méconnaissance presque totale a paradoxalement produit chez les habitants tout un ensemble de représentations fabulées construites autour du caractère maléfique attribué au volcan, perçu au début du XVIIIe siècle comme le pays «consumé par les feux souterrains» d'où l'on a peu de chance de revenir, du moins si l'on tente d'en atteindre le sommet. De fait, la difficulté du voyage réside moins dans la malfaisance imaginaire du volcan que dans les rudes conditions géographiques imposées aux visiteurs de la Fournaise. La contrainte météorologique apparaît la plus importante, car elle conditionne la réussite de l'entreprise. Elle s'exprime par des risques de très fortes précipitations (Robert, 2003) ${ }^{3}$ et par de brusques changements météorologiques entraînant une chute rapide des températures. Amplifiées par l'altitude du massif du volcan (sommet à $2600 \mathrm{~m}$ ), au cours de l'hiver austral, les valeurs de température affichées par le thermomètre sont fréquemment négatives ${ }^{4}$. Il est ainsi compréhensible que les excès de ce facteur soient ceux qui retiennent le plus l'attention des visiteurs.

Caractérisée par de profondes vallées et par des effondrements caldeiriques importants, dont témoignent les remparts et les enclos ${ }^{5}$ successifs, la configuration du relief de la Fournaise ajoute sa part de difficulté au projet. Enfin, l'état indigent du réseau de communication, depuis l'origine du peuplement jusqu'aux années 1950 (Defos du Rau, 1960: 571-574) ${ }^{6}$, réduit considérablement les déplacements souvent assimilés à de véritables odyssées. C'est de ce contexte géographique très singulier que procède en grande partie la fabrication d'itinéraires permettant l'accès au bord du volcan (figure 1).

3 Les travaux de René Robert ont bien mis en évidence l'importance des précipitations sur le massif du volcan dont les valeurs médianes sont généralement supérieures à $12000 \mathrm{~mm}$ par an. En soulignant qu'aux moments des grandes perturbations tropicales, «tous les records mondiaux de pluviométrie entre 12 heures et 15 jours consécutifs sont détenus par La Réunion", notre collègue note "plus de 6000 mm en quinze jours consécutifs au cratère Commerson en janvier 1980» (Robert, 2003 : 26).

4 Cette rigueur du climat est soulignée dans chacun des récits du voyage au volcan réalisés entre le mois de mai et la fin d'août. La présence «d'une glace qui pousse en terre», selon l'expression imagée des guides, est la figure récurrente que les curieux matinaux découvrent au départ de la caverne des Lataniers (alt. $2390 \mathrm{~m}$ ). Les épisodes neigeux ne sont pas rarissimes à cette altitude comme le prouvent les chutes notables (entre 3 et $5 \mathrm{~cm}$ ) du mois d'août 2003, celles de septembre 2007 et de juin 2013 .

5 À La Réunion, ces deux termes désignent principalement les bordures et les fonds des grands effondrements du Piton de la Fournaise. Ces figures morphologiques matérialisent les caldeiras, qui sont des structures très communes dans les volcans boucliers du volcanisme intraplaque.

6 Auteur de la première thèse de géographie sur La Réunion, Defos du Rau signale qu’en 1946 «la partie la plus efficace du réseau routier se réduit à $131 \mathrm{~km}$ de voies bitumées sur les $736 \mathrm{~km}$ ", le reste étant en très mauvais état et subissant des coupures fréquentes à la période des grosses pluies (1960: 571-574). 
Figure 1 Accessibilité et itinéraires potentiels du Piton de la Fournaise

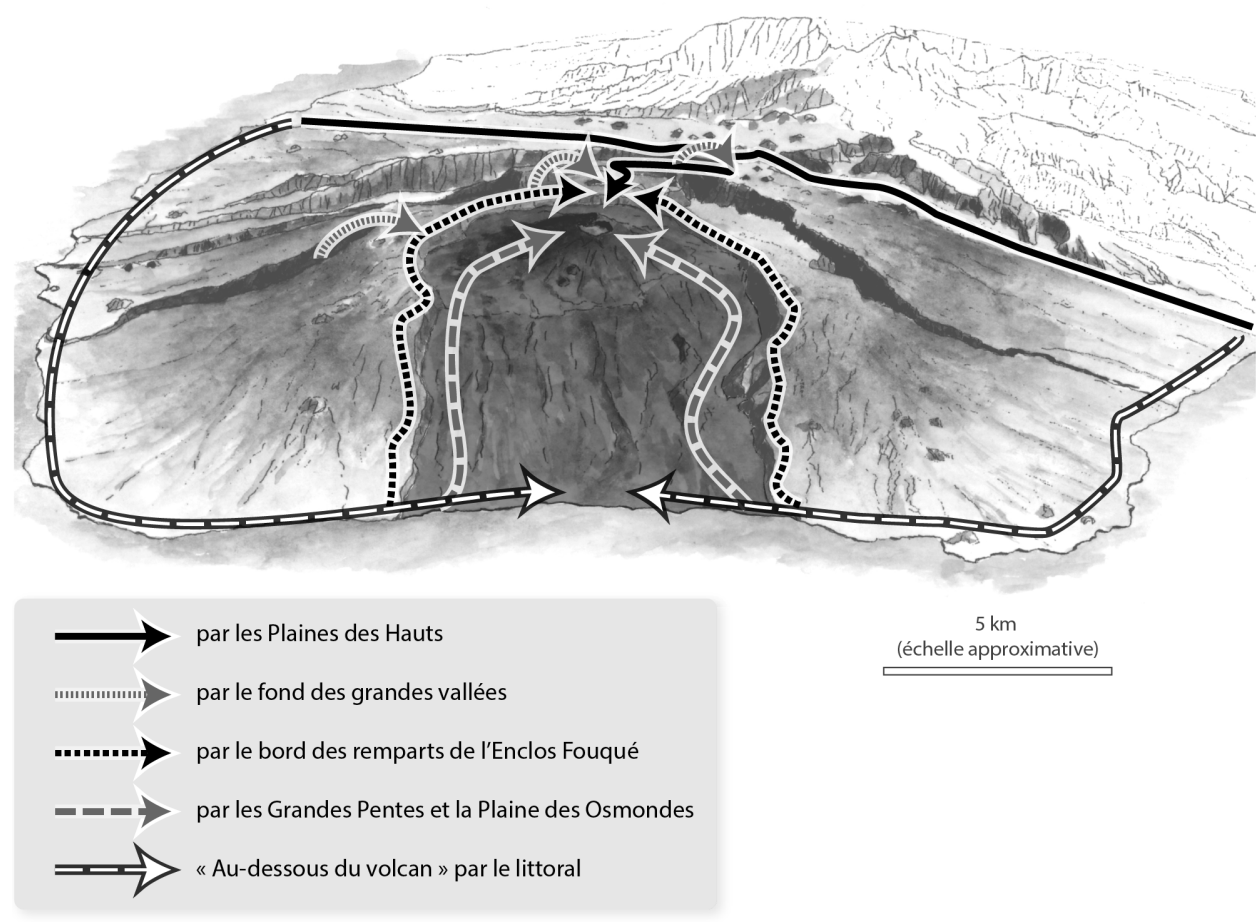

Jusqu'à la fin du XIXe siècle, l'itinéraire par le littoral a été le plus utilisé pour se rendre sur les éruptions ou simplement pour " passer » au Volcan. Mais certains visiteurs, plus curieux, ont souhaité assez tôt (milieu du $\mathrm{XVIII}$ siècle) atteindre le sommet du Piton de la Fournaise. Pour mener ce projet, plusieurs options ont été expérimentées: la remontée des Grandes Pentes et de la Plaine des Osmondes, mais le terrain chaotique des " coulées grattons » rend le cheminement compliqué et pénible; l'accès par les bords de l'Enclos n'a pas été plus facile, à cause de la présence d'une végétation primaire assez inextricable jusque vers $1800 \mathrm{~m}$ d'altitude et en raison d'un temps souvent pluvieux. Les itinéraires par le fond des grandes vallées ont été parcimonieusement empruntés pour des questions de logistique et d'accessibilité. Le somin Volcan par les Plaines des Hauts est devenu assez vite la solution de confort et de sécurité pour réussir le voyage au volcan.

Conception et réalisation: Ch. Germanaz et M. Sicre

Adapté par le Département de géographie de l'Université Laval

\section{L'itinéraire et sa pérennité}

L'attraction des sommets du massif volcanique pour les chasseurs de fouquets ${ }^{7}$ et de cabris, la préoccupation vitale de trouver un refuge inaccessible pour les marrons ${ }^{8}$ et l'insatiable curiosité de plusieurs résidants entraînent assez tôt la présence ponctuelle des hommes sur les bordures des remparts qui structurent la partie haute du volcan. Les empreintes discrètes qu'ils ont laissées de leurs premières incursions sont enregistrées dans la tradition orale sous la forme d'un canevas cartographique confidentiel constitué de sentes à peine visibles et de sentiers éphémères. Ce dépôt mémoriel a guidé les visiteurs suivants dans leur choix d'un itinéraire susceptible

7 Le fouquet est un oiseau marin endémique de La Réunion, connu plus communément sous le nom de pétrel de Bourbon (Pseudobulweria aterrima).

8 Qualificatif attribué aux esclaves en fuite. 
d'assurer leur conquête du volcan dans des conditions de sécurité et de praticabilité acceptables. Si les accès "au-dessous du volcan» n’ont pas posé de difficultés insurmontables, l'accession aux enclos sommitaux a été une tout autre aventure. Plusieurs options ont été expérimentées au cours des voyages originels, dont certaines ont d'ailleurs perduré jusqu'au début du XXe siècle. Emprunté dès l'origine, c'est le chemin des plaines, dont la Plaine des Cafres, qui finit par rallier la préférence des voyageurs (figure 1). En effet, la sûreté et le confort relatifs procurés par la présence de plusieurs cavernes pour les bivouacs, un contournement judicieux des obstacles topographiques les plus pénibles et une optimisation empirique du tracé pour découvrir les paysages les plus spectaculaires assurent au somin Volcan la qualité de «meilleur itinéraire» (Ozoux, 1938: 184-186) pour se rendre à la Fournaise (figure 2). En reprenant l'essentiel du trajet, la réalisation d'une route forestière au début des années 1960 consacrera la pérennisation de ce choix.

\section{La fabrication du mythe}

La singularité de l'environnement géographique du voyage à la Fournaise n'explique pas à elle seule, la résonance profonde que provoque toujours l'évocation du somin Volcan dans l'imaginaire des vieux habitants de l'île. Longtemps considérées comme une étrange aventure, les visites à la Fournaise et ses épisodes les plus héroïques ont été facilement appropriés par la mémoire collective. Si cette mémoire ne restitue pas une image complète de chaque visite, elle intègre toujours une part de fascination et d'effroi associée aux récits du somin Volcan. Comprendre l'enchantement de cet itinéraire nécessite donc de démêler «l'écosystème» complexe de sa mythification.

La dimension sociétale participe à ce système par l'architecture sociale des voyages au volcan, qui traduit assez justement les stratifications existant à l'intérieur de la société créole des premiers temps de l'île. La composition des voyageurs se subdivise schématiquement en trois groupes : les porteurs, les guides et les visiteurs, ces derniers étant généralement à l'origine de l'aventure. Des extraits de dessins et de photographies couvrant la période 1791-1964 permettent d'identifier chaque fois le trio d'acteurs associé durablement dans le voyage au volcan (figure 3).

Jusqu'au milieu du XIXe siècle, les esclaves forment le groupe des porteurs. Ceux-ci sont ensuite recrutés parmi les affranchis, les engagés (excursion de Charles Vélain en 1874) et, au siècle suivant, les «Petits Blancs» des Hauts. Originaires des basses pentes du volcan entre Saint-Benoît et Saint-Pierre et plus tardivement de la Plaine des Cafres, les guides sont mieux identifiés, car leurs noms apparaissent clairement dans les récits (Germanaz, 2005a: 96). Ils sont engagés pour leurs bonnes connaissances du terrain. Quant aux visiteurs, natifs de l'île, résidants de longue durée ou voyageurs de passage, ils présentent sommairement trois profils: ils sont administrateurs (puis fonctionnaires), scientifiques (naturalistes, géologues, géographes) ou plus simplement des passionnés du volcan. Soudé momentanément par l'entreprise, ce trio social partage les rudes conditions du voyage. 


\section{Figure 2 Le somin Volcan, les étapes du meilleur itinéraire}

\section{Étape 1: de la Plaine des Cafres au Bras de Pontho}

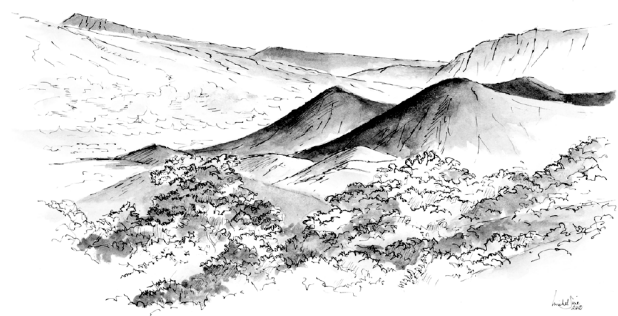

Le départ du somin Volcan s'effectue du Piton Villers à la Plaine des Cafres. L'entreprise commence par un

cheminement dans une petite ravine, le Bras de Pontho. L'itinéraire offre une vue générale sur la Plaine et sur les Pitons adventifs du massif de la Fournaise.
Étape 2: de la Rampe Zézé à la caverne des Lataniers

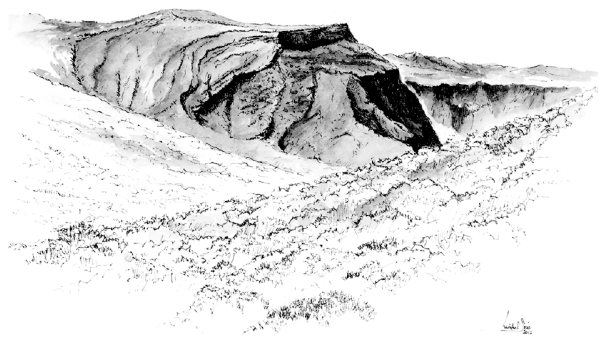

A la sortie du Bras de Pontho, les voyageurs accèdent à la Plaine des Remparts et à la caverne des Lataniers par une dernière pente toujours éprouvante, la rampe Zézé. Ce toponyme reprend le petit «nom gâté » d'un porteur qui aurait glissé dans les scories.

\section{Étape 3: le bivouac à la caverne des Lataniers}

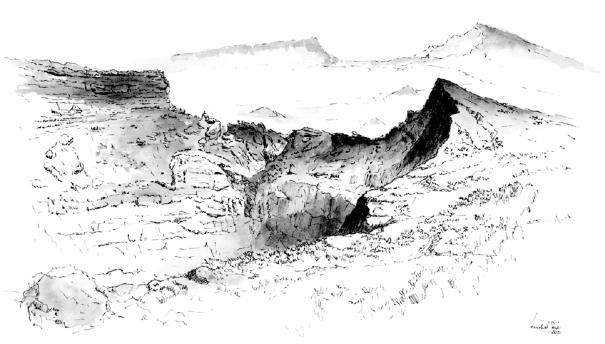

La vue sur le cratère Commerson et le Piton des Neiges

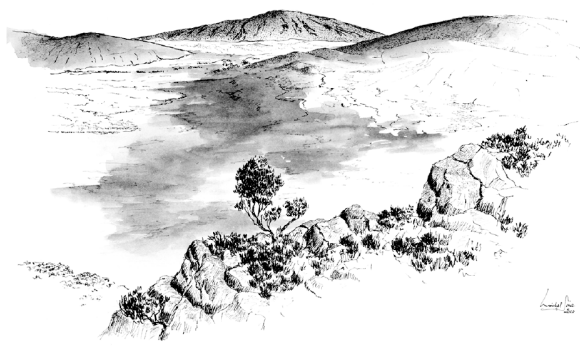

La vue sur la Plaine des Sables depuis l'Oratoire Ste-Thérèse

La halte nocturne à la caverne permet aux visiteurs de contempler deux points de vue emblématiques du voyage au volcan, le premier sur le cratère Commerson et le second sur la Plaine des Sables à partir duquel ils peuvent également apercevoir le Piton de la Fournaise pour la première fois dans l'excursion.

\section{Étape 4: I'arrivée au Pas de Bellecombe}

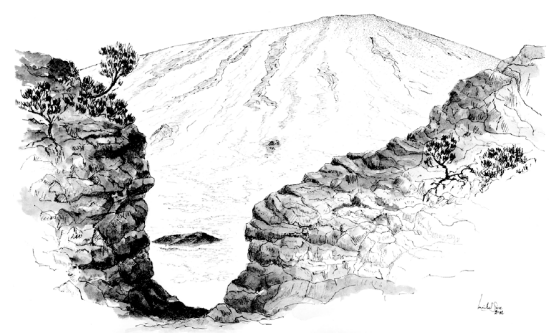

Le voyage au volcan s'arrête au bord de l'Enclos Fouqué pour la plupart des voyageurs, entre 1750 et 1964. La vue depuis ce point permet de découvrir la vaste caldeira dans laquelle se situent le petit cratère du Formica Leo ( $2^{\mathrm{e}}$ plan) et le majestueux Piton de la Fournaise (arrière plan).

Conception et réalisation: Ch. Germanaz et M. Sicre
Étape 5 (facultative): le sommet du Piton de la Fournaise

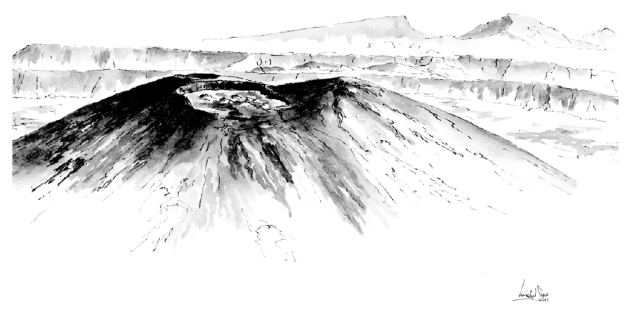

Les plus intrépides tentent l'ascension du Piton et découvrent les cratères sommitaux dont la morphologie évolue au fur et à mesure des éruptions (ici la reconstitution correspond aux années 1750). Au second plan, le système des caldeiras emboîtées et en arrière-fond, le Grand Bénard (gauche) et le Piton des Neiges (droite). 
Figure 3 Architecture sociale des voyages au volcan (1790-1950)

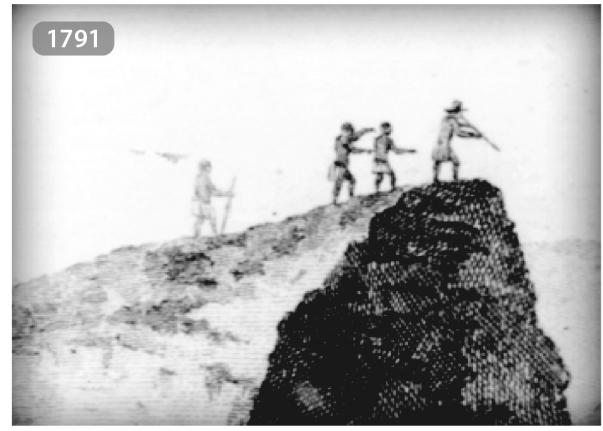

Illustration extraite du texte de Philippe Petit-Radel relatant sa visite du volcan en 1791 .

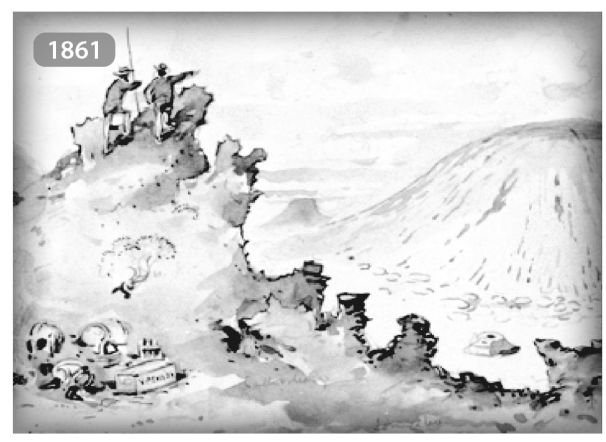

Scène de l'aquarelle du Marquis de Trévise, le volcan de Bourbon (1861).

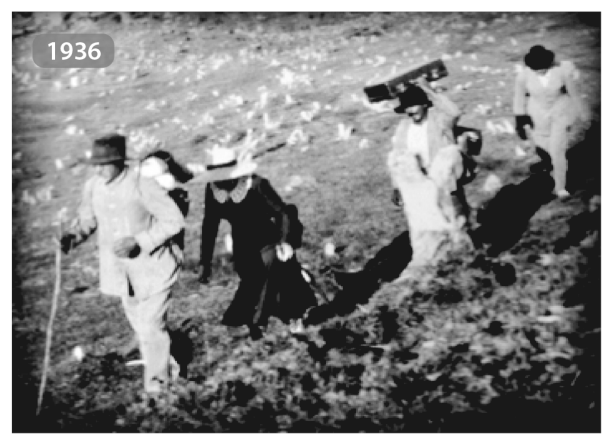

Photo de la collection privée H. Cornu ( 1936).

Conception et réalisation: Ch. Germanaz

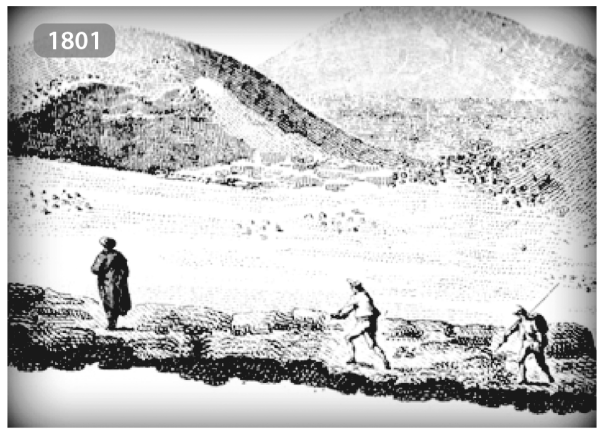

Extrait de la gravure de l'ouvrage de Bory de Saint-Vincent sur son passage à la Fournaise en 1801.

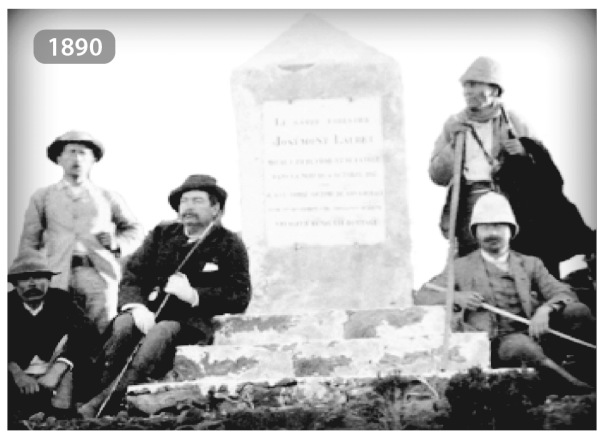

Photographie de la collection H. Georgi (1890), Archives départementales de La Réunion.

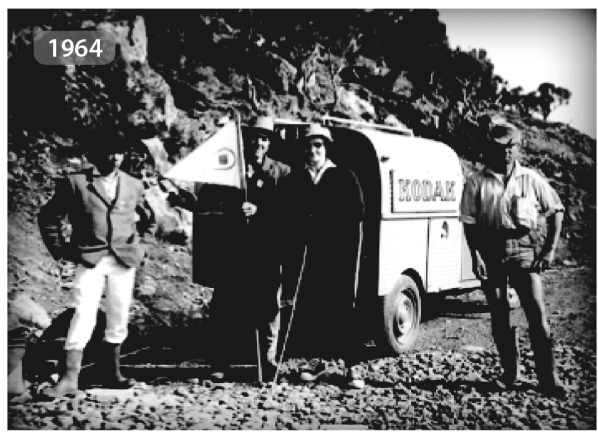

Cliché A. Blay, collection M. Blay ( 1964).

Adapté par le Département de géographie de l'Université Laval

Cette solidarité de fait est vécue le plus souvent, de manière symbolique, comme une réunification temporaire de tous les fragments de la société coloniale, rappelant le temps idéalisé des premiers arrivants où les barrières sociales n'étaient pas encore édifiées. De la même façon, ce temps idéel des origines du peuplement de l'île s'impose aux voyageurs par la pratique des bivouacs dans les grottes ou les cavernes qui balisent l'itinéraire. Cette manière d'habiter le volcan facilite la mise en scène d'un retour au 
temps originel à la faveur du repas du soir où, à la lumière du feu de bois, les zistwar lontan ${ }^{9}$ prennent une profondeur incantatoire. Le temps des récits s'impose alors aux visiteurs qui renouent avec la geste légendaire des chasseurs de cabris et celle, plus douloureuse, du marronnage et de sa répression. Si, d'aventure, un cri de fouquet perce à l'intérieur de la caverne, le conteur s'interrompt et, sans la nommer, chacun évoque en frissonnant Gran mér Kal10. L’association de ces récits épiques et des légendes noires participe à l'enchantement du somin Volcan et à la construction d'un "entre-temps", parenthèse momentanée entre le tan lontan et celui des contemporains, favorable à une contemplation méditative face aux vastes panoramas des paysages de la Fournaise.

Conducteur du regard, le somin Volcan est ponctué par quatre ou cinq points de vue paysagers majeurs dont l'observation organise de manière invariable le rythme du cheminement et l'ordre des haltes. Sans reprendre ce que nous avons déjà développé dans d'autres publications sur cet aspect important des voyages au Piton de la Fournaise (Germanaz, 2004, 2009 et 2013), nous devons insister sur ce sentiment indicible éprouvé et partagé par l'ensemble des "hommes aux semelles volcan» face au spectacle «effroyable et sublime» de la nature volcanique. Cette perception est l'une des substances très actives du voyage, favorisant de manière significative la gestation du mythe (Germanaz, 2005b). L'analyse de la plupart des récits de cette période met en évidence des perceptions de nostalgie et des épanchements de romantisme face l'imposante grandeur des paysages. L'exemple de l'étape de la caverne des Lataniers est, à cet égard, véritablement emblématique des sentiments éprouvés le long du somin Volcan. Les textes exposent une figure récurrente présentant les curieux, entre chien et loup, contemplant silencieusement l'immensité troublante de la Plaine des Sables sous l'ombre des derniers rayons du soleil ou observant d'un regard inquiet la profondeur obscure du cratère Commerson dans une lumière bleutée crépusculaire. Scientifiques, visiteurs enclins à la poésie ou amateurs éclairés, tous évoquent une impression de vertige assénée par la confrontation de la dimension humaine à celle des paysages du volcan. Cette sensation les réunit dans une forme d'expérience extatique qui participe à la renommée du somin Volcan.

Le texte passionné du docteur Louis Ozoux (1938), à propos de son excursion au volcan en 1928, est sans doute l'un des meilleurs témoignages de cette posture. Voici la description du paysage que fait l'homme de l'art en découvrant le volcan au petit matin:

\footnotetext{
[...] et voici que dans la brume grise, presque au sommet du Piton qui est d'un beau violet sombre, apparaît une sphère rouge, une lanterne vénitienne suspendue au ciel par un invisible fil! Le soleil! Quelle scène! Tout l'être se crispe et se dilate tour à tour ; on rit de joie; on se tait de respect; on demeure confondu de ce que tant de choses affreuses puissent être enveloppées de tant de charme (Ibid. : 196).
}

Plus loin, l'auteur constate:

\begin{abstract}
Cette heure a été pour nous, l'heure idéale [...] jamais personne n’aura spectacle plus rare; pour les yeux, mélange des clartés du matin et des ténèbres de la nuit, de teintes fraîches et de tons sinistres, de formes séduisantes et de lignes rébarbatives; pour l'âme, l'étonnement,
\end{abstract}

9 Cette expression créole, qui peut s'écrire également sous la forme zistoir lontan, désigne l'ensemble des récits anciens (les histoires du temps longtemps) qui participent à la construction de l'identité réunionnaise.

10 Gran mér Kal est une figure emblématique des légendes noires de La Réunion. Elle est représentée sous les traits d'une vieille sorcière dont on menaçait autrefois les enfants capricieux. Si elle peut «rôder» dans les ravines, elle affectionne plus particulièrement le Piton de la Fournaise. 
la volupté, la crainte tout ensemble; au fond de moi c'est la sensation héroïque, la perception de la force, de la sauvagerie primitive, de la fantaisie furieuse de cette Nature capable d'une catastrophe immédiate, cassant l'île en deux, en dix, l'engloutissant avec ses habitants, sa société, sa civilisation; pas même quelques écueils à sa place! (Ibid. : 196).

Le ton est encore plus élégiaque et emphatique lorsque, au moment de quitter les cratères sommitaux, le médecin de la colonie, saisi par une sorte d'ivresse des sommets, adresse au volcan un dithyrambe ambigu:

Salut donc à toi, Piton de la Fournaise [...] Ta forme est à la fois celle d'un exquis sein de vierge et celle d'une abominable pustule; on te montre le poing et l'on ne cesse de t'adorer.

[...] Source de passion, poison dont on veut toujours davantage, quitte à en périr, tu hantes nos jours et nos songes [...]

Pour les joies légères, pour les enthousiasmes qu'ont soulevés en moi les sentiers fleuris, mer de sable, dôme incandescent, fleuves de lave, gouffre d'enfer, sois remercié! (Ibid. : 212-213).

La tonalité du récit de Louis Ozoux n’apparaît pas comme un objet singulier à l'intérieur du corpus textuel des voyages au volcan, certains visiteurs (Jacob de Cordemoy, 1862 ; Héry, 1883 ; Hermann, 1890) vont même jusqu'à exalter en direction de leurs contemporains la valeur hautement hygiéniste (physique et morale) de l'excursion. L'itinéraire qui lui donne naissance reste, tout au long de l'édification du mythe, un formidable parcours initiatique. À peine entamée par les pratiques nouvelles qui s'installent progressivement chez les visiteurs à la fin des années $1920^{11}$, cette propriété s'évapore au moment de la massification des visites de la Fournaise, provoquant alors une banalisation paradoxale du somin Volcan.

\section{La perte des récits ou la banalisation du somin Volcan}

Les données liées à cette seconde période sont issues du traitement d'un ensemble d'enquêtes réalisées par l'ONF (souvent inédites comme le comptage de la fréquentation de la route du volcan effectué en 2002) ou dans le cadre de mémoires de maîtrise, de master et de thèse dirigés au sein du département de géographie de l’Université de La Réunion (Avice, 2001 ; Payet, 2005; Jacquard, 2009; Morin, 2012) ou de celui de l'Université Montpellier (Mas, 2012). Elles sont enrichies par des entretiens personnels avec certains acteurs du voyage au volcan (les familles Cornu, Gérard, Blay, Albany, les anciens guides, porteurs et gardiens du gîte de Bellecombe). Depuis plus de 10 ans, l'organisation quasi annuelle d'une sortie de terrain sur le massif de la Fournaise pour les étudiants de géographie nous a permis de dresser un tableau assez précis de leurs représentations et de leurs connaissances spatiales du volcan. Celles-ci formalisent l'héritage implicite des perceptions relatives à la visite du volcan d'une partie de la population.

Au milieu des années 1960, la séduction de la visite à la Fournaise échappe au cercle des initiés pour conquérir un public plus large. Exprimée par une augmentation exponentielle du nombre des curieux au bord du volcan, la rapidité de cette conquête, au-delà de la notoriété attractive dont jouit désormais le phénomène volcanique, est à mettre en relation avec l'ouverture d'une route forestière, l'établissement d'un nouveau gîte permanent au bord de l’Enclos, l'amélioration générale du réseau de circulation, la

11 Vers 1930, les cavernes ne sont presque plus utilisées comme havres nocturnes par les visiteurs. 
diffusion de l'automobile et un nouvel intérêt pour la randonnée dans les Hauts de l'île (Bouchet et Gay, 1998). La massification des flux de visiteurs met fin à la conception et à l'esprit du voyage au volcan, provoquant un désenchantement du somin Volcan dont la banalisation n’a cependant pas produit tous les aspects négatifs que le terme présuppose.

\section{La massification des flux de visiteurs}

L'amélioration des conditions du voyage au Volcan débute au cours des premières décennies des années 1900. Pressé par le gouverneur Jules Repiquet, le syndicat d'initiative de La Réunion, en collaboration avec le Service des Eaux et Forêts, réalise en 1927 un chemin de cavaliers de la Plaine des Cafres au Pas de Bellecombe. Pour sécuriser l'excursion, qui dure encore trois jours au minimum, deux gîtes «bâtis en pierre, [et] couverts de bardeaux» (Merwart, 1929) sont aménagés. Détruits moins d'un an plus tard par le cyclone de 1928 mais reconstruits plusieurs fois entre 1930 et 1939, ces dispositifs n'ont pas augmenté sensiblement le nombre des curieux, le somin Volcan exigeant toujours une pratique pédestre. Moins fréquentées par les visiteurs, les cavernes naturelles restent cependant un abri de choix pour les quelques chasseurs occasionnels, pour les guides et les porteurs qui "préfèrent aller à la caverne pour faire du feu, rire et causer à leur aise» (Ozoux, 1938: 201). Sans remettre profondément en cause la dimension initiatique de l'excursion, cette évolution des pratiques modifie certains tracés de l'itinéraire qui, selon la décision du guide, peut emprunter désormais le Pas des Sables pour rejoindre les bords de la Fournaise.

La construction d'une route forestière ouverte à l'automobile, reliant la plaine des Cafres au bord de l'Enclos, bouleverse totalement l'esprit et la logistique du voyage au volcan. Construite non sans difficulté entre 1957 et $1972^{12}$, cette route reprend les grandes lignes du somin Volcan et réduit considérablement le cheminement pédestre de l'entreprise, toujours incontournable pour atteindre le sommet de la Fournaise. Elle transforme donc l'expédition en une excursion moins sélective et installe progressivement le volcan comme l'un des hauts lieux touristiques de l'île. Cette infrastructure au succès quasi immédiat canalise, aujourd'hui, un afflux de visiteurs estimé à plusieurs centaines de milliers par ${ }^{13}{ }^{13}$. Invitée au volcan, l'automobile a pris une place débordante au bord de l'Enclos et, lors des éruptions majeures, il n'est pas rare d'observer de véritables embouteillages (1998). Si ces flots envahissants impriment incontestablement leurs marques sur l'environnement du massif volcanique, ils ont surtout vidé le somin Volcan de toute sa substance épique et déstructuré sa dimension sociale en faisant disparaître les guides et les porteurs.

12 Le début des travaux fut plutôt rapide puisque, au bout de deux ans (1959), le Nez de Bœuf était atteint. Il a fallu ensuite sept ans pour rejoindre le cratère Commerson (1966). En atteignant le bord de l'Enclos, au début de l'année 1968, la route ouvrait désormais l'accès du volcan à la plupart des curieux. Les travaux d'enrobage sont achevés en 1972 pour la plus grande partie du trajet et en 1992 pour l'ensemble, hors Plaine des Sables. En 2004, les $16 \mathrm{~km}$ de la route forestière ont été totalement rénovés pour assurer leur intégration à l'environnement volcanique.

13 On peut estimer à moins d'un millier le nombre de voyageurs à visiter les hauts du volcan entre 1750 et 1965 (Germanaz, 2005a). Pour l'année 2011, les comptages réalisés par l'ONF et le parc national établissent à 450000 le nombre des visiteurs à l'entrée de la route forestière et à un peu plus de 340000 ceux qui se rendent au bord de l'Enclos. 


\section{Le désenchantement du somin Volcan}

Le phénomène n’est pas particulier à La Réunion. Il reste assez conforme à la «biologie» des itinéraires culturels qui, après une phase de fabrication, ont presque tous connu une étape de déconstruction à une période plus ou moins identique de leur histoire. Entreprise à partir des années 1960, cette déconstruction est liée à leur valorisation touristique ou, plus simplement, à une modernisation de leur accès en faisant de l'infrastructure routière une priorité pour favoriser la consommation massive des sites remarquables. L'introduction de l'automobile bouleverse le sens de la piste, du chemin ou de la route traditionnelle. Il s'agit dorénavant de rallier rapidement des étapes, des destinations, en effaçant du même coup la temporalité et la géographicité de ce qu'on pourrait appeler les entre-deux, c'est-à-dire ces intermèdes d'espace-temps entre les lieux visités, nécessaires pour faire émerger une relation d'intimité entre le voyage et le voyageur et propices à l'enchantement de l'itinéraire. La compression du temps, l'effacement des repères spatiaux originels et la disparition des grands récits aboutissent ainsi au désenchantement des chemins de culture.

Pour celui de la Fournaise, la compression du temps est celle de la durée de l'excursion, réduite par la route forestière à quelques heures ${ }^{14}$ au lieu des trois jours initiaux. Elle est aussi une accélération du temps qui, en obligeant les curieux à une mobilité plus rapide et à une sélection des points de vue, évacue toute probabilité d'expérience initiatique. La route du volcan a conservé du somin Volcan trois haltes plus ou moins décalées de leur situation d'origine. Entre ces lieux de vue (Nez de Bœuf, cratère Commerson et Plaine des Sables) qui formatent le regard des touristes, s'étend le vide engendré par la perte des grands récits effacés de l'itinéraire. La stèle commémorant la mort, en 1887, du guide Josémont Lauret (et de son neveu), héros d'une épopée fatale pour sauver ses compagnons, est à l'écart du chemin de l'automobile. Les tragédies de l'histoire du marronnage sont occultées aux yeux des visiteurs dans un espace informel. La disparition de la compagnie des guides et des porteurs entraîne celle de la transmission des récits. Aujourd'hui, les avatars de ces derniers se résument le plus au fait divers: touristes égarés par le brouillard ou morts de froid par imprudence...

Ainsi, la déconstruction du somin Volcan par l'effacement de l'itinéraire originel et son corollaire, l'occultation des repères spatiaux symboliques (cavernes, grottes, oratoires), contribuent à un lissage de la mémoire des lieux et à la reconstruction d'une histoire normative, focalisée sur l'attraction de la Fournaise.

\section{Une banalisation paradoxale}

La banalisation qui s'ensuit est paradoxale, car elle transforme l'itinéraire du volcan en un lieu commun pour le touriste tout en provoquant son appropriation culturelle par la population locale. Cette transformation est liée à une sublimation de l'objet volcanique, apprivoisé et médiatisé depuis la fin des années 1960. Son regain d'attraction auprès des visiteurs et l'existence de la route forestière diminuent la séduction de l'étape intermédiaire, le but pour l'ensemble des curieux étant prioritairement d'atteindre le bord de l'Enclos et, si le temps disponible le permet, de s'arrêter aux trois points

14 Il faut en moyenne de 30 à 40 minutes pour atteindre le Pas de Bellecombe depuis l'entrée de la route forestière. Avec arrêts aux trois points de vue majeurs de l'itinéraire, il faut ajouter 20 ou 30 minutes selon les visiteurs. Le tour des cratères sommitaux nécessite trois heures supplémentaires. 
de vue institutionnalisés par les voyages au volcan. Mais la curiosité pour la route du volcan ne disparaît pas complètement, elle est renouvelée par des installations qui proposent une lecture différente des paysages livrés à l'observation des touristes. Au temps de la découverte contemplative succède celui de l'explication.

En effet, la massification des flux de visiteurs décide les services de l'État, l'ONF en premier, à réaliser une mise en tourisme de l'itinéraire du volcan. Le parti pris de cet aménagement touristique repose sur l'idée «d'expliquer la Fournaise» aux visiteurs par la médiation d'une lecture commentée de ses paysages. La matérialisation de cette pédagogie se traduit par la création, aux étapes «historiques» de la route du volcan, d'un espace public structuré invariablement en trois éléments: l'aire de stationnement avec sa poubelle, le belvédère et son mobilier (table d'orientation, panneaux explicatifs sur le thème du paysage observé et sur les formations végétales environnantes...) et, en coalescence avec ces derniers, une aire de loisirs limitée où l'on trouve parfois un kiosque invitant à prolonger l'arrêt. La standardisation de cet aménagement, observable à l'échelle du territoire métropolitain, contribue à la banalisation d'une découverte dont le contenu scientifique, bien que simplifié, ne retient pas toujours l'attention des visiteurs. Les dessins des morphologies et des dynamiques volcaniques du Piton de la Fournaise captent bien l'attention des touristes, mais la lecture des explications qui les accompagnent s'interrompt la plupart du temps très rapidement ${ }^{15}$. Quant à la poubelle, elle est très souvent débordée par un flot de déchets qui contraste violemment avec la naturalité affichée de ces points de vue remarquables.

L'aspect positif de la banalisation du somin Volcan a été de permettre aux habitants de l'île de s'approprier complètement le volcan. Les manifestations de cette appropriation sont nombreuses. C'est déjà, dans le langage courant, l'apparition de l'expression Not'Volcan (notre volcan) pour accompagner la désignation du Piton de la Fournaise. C'est ensuite sa mobilisation fréquente comme figure métaphorique par les hommes politiques de l'île pour dénoncer la fissuration des solidarités de la société réunionnaise et pour sonner l'alarme sur les risques d'effusion d'une population en apparence passive (le volcan endormi), mais confrontée jour après jour à une dégradation alarmante de la situation socioéconomique. C'est enfin, de manière encore plus visible, la présence dominicale d'une véritable ligne de pique-niqueurs, presque ininterrompue, de l'entrée de la route forestière jusqu'aux environs de la Plaine des Sables. L'accès au crédit pour l'achat d'une automobile, généralisé à l'ensemble de la population, a permis l'élargissement géographique des parcours de loisir et, plus largement, le développement d'un des traits culturels fondateurs de l'identité réunionnaise: le besoin quasi vital de se retrouver régulièrement immergé en pleine nature, renouant par là même, plus ou moins consciemment, avec le temps des origines; ce tan lontan où la population trouvait l'essentiel de ses ressources dans l'exploitation, souvent excessive, d'une nature généreuse dont l'abondance des fruits semblait illimitée. Ainsi, peu enclins à rester face à leur téléviseur, les habitants préfèrent chaque dimanche, voire sur la totalité de la fin de semaine, investir les espaces emblématiques de l'île. Dans ce panthéon, le volcan tient une place de

15 Ce constat reste empirique, il procède de l'observation quasi mensuelle, sur une période de plus de 10 ans, des visiteurs lors de ces arrêts "pédagogiques». Plus étonnant, au cours des excursions annuelles avec les étudiants de géographie, nous avons constaté que $80 \%$ de ces visiteurs n'allaient pas au bout de leur lecture des panneaux. 
choix, non seulement par la qualité du «changement d'air » 16 qu'il occasionne, mais surtout par l'imaginaire et la convivialité qui lui sont associés. Cette tradition récente revendiquée par les habitants exprime une forme de résilience identitaire du somin Volcan dont la patrimonialisation actuelle caractérise la période contemporaine.

\section{Le retour au récit et la patrimonialisation du somin Volcan}

Ce dernier temps repose sur le dépouillement des inventaires, des rapports et des enquêtes publiques nécessaires à la création du parc national de La Réunion et à la candidature pour son inscription au patrimoine mondial. Ce travail a été coordonné, pour l'essentiel, par notre collègue René Robert (2006, 2009). Les stratégies d'interprétation et de valorisation écotouristique de la route du volcan analysées dans cette partie ont été proposées par Michel Sicre à l'ONF puis dans le cadre de sa mission au parc national. Il n'existe pas encore de statistiques spécifiques sur la fréquentation du massif volcanique, mais on peut toujours obtenir quelques éléments d'appréciation (flux et ressentis sur l'excursion) en interrogeant directement les responsables de l'IRT (Île de La Réunion Tourisme), de l'ONF et de l'Observatoire régional du tourisme.

À partir de la fin des années 1990, la Région Réunion accentue la réflexion qu’elle avait engagée sur deux fronts : la préservation et la valorisation des ressources fauniques et floristiques endémiques et la promotion touristique des sites les plus spectaculaires de l'île (candidature à l'inscription de ses pitons, cirques et remparts au patrimoine de l'humanité). Ce regain d'intérêt pour cet héritage naturel et culturel suit un mouvement général à l'œuvre dans l'ensemble des pays occidentaux et formalisé par des politiques de patrimonialisation tous azimuts en réaction probable aux effets de standardisation induits par une globalisation économique triomphante. Appliquées à La Réunion, ces politiques aboutissent à la création d'un parc national (2007) et à l'inscription de ses territoires de caractère au patrimoine mondial (2010). Ce nouveau contexte institutionnel offre l'occasion d'une revalorisation du somin Volcan et de certaines pratiques le caractérisant comme le retour des guides Péi et celui du cheminement pédestre en diversifiant les offres de randonnée autour de l'itinéraire.

\section{Nouveau contexte et nouveaux récits}

En multipliant le nombre des visiteurs entre les années 1970 et 1990, la banalisation de la route du volcan a produit une pression visible sur l'environnement du massif volcanique. La saturation rapide des sites d'accueil aux étapes intermédiaires et au bord de l'enclos Fouqué 17 a conduit les curieux à ouvrir des aires de pique-nique sauvages dénuées d'équipements et de protection contre les sinistres. Or, la spécificité de la végétation altimontaine du volcan (landes à érica, fourrés de sophora et acacia, tous très

16 À La Réunion, le «changement d'air» est une tradition qui consiste à «monter» dans les Hauts de l'île (hautes plaines et cirques) au moment des grosses chaleurs estivales pour bénéficier des températures plus clémentes liées à l'altitude. Attesté dès le milieu du XIXe siècle pour Salazie, le "changement d'air» se généralise au cours du siècle suivant. L'amplitude thermique entre le littoral et le bord de l'Enclos, au Pas de Bellecombe, est souvent supérieure à $10^{\circ} \mathrm{C}$.

17 L'enclos du Pas de Bellecombe a été nommé au début du XXe siècle du nom du géologue Ferdinand Fouqué (1828-1904) dont les travaux ont porté, en partie, sur les manifestations volcaniques. Marié à la fille du scientifique, c'est Alfred Lacroix (1863-1948) qui, lors de son passage en 1911, serait à l'origine du toponyme. Peu utilisé localement, on lui préfère le plus souvent le terme simplifié d’Enclos. 
inflammables) et les périodes de sécheresse successives constituent une conjonction favorable au feu. Ces facteurs sont la plupart du temps ignorés par l'ensemble des touristes. Plusieurs grands incendies (1994, 2010 18) ayant pris naissance à proximité de la route ont dévasté les hauts du massif sans qu'il soit possible, aujourd'hui, d'en attribuer l'origine de manière formelle aux visiteurs du volcan. Nous pouvons rappeler que l'incendie sur le massif de la Fournaise n'est pas un événement nouveau; lors de son passage au volcan, en 1928, Louis Ozoux signalait, à propos du Nez de Bœuf:

\begin{abstract}
Ses pentes sont brûlées depuis 1904; sur le fond charbonné, troncs et branches, fanés par la pluie et l'air, ont des blancheurs d'ossements humains et sont aussi pitoyables; il y en a un nombre formidable; pourquoi ne pas les exploiter? Le guide va nous le dire: «Ne leur donnez pas ce bois, ils mettront de suite le feu ailleurs pour avoir d'autres bois (Ozoux, 1938: 188).
\end{abstract}

Dans l'attente des résultats de recherches récentes sur l'origine de ces sinistres et de la production d'une cartographie historique de l'aléa «incendie», les responsables du parc national et de l'ONF constatent empiriquement une augmentation de leur fréquence parallèlement à celle des visiteurs du volcan, mais aussi à la faveur des changements climatiques ${ }^{19}$. C'est pourquoi le choix des pouvoirs publics s'est porté sur la délimitation et l'aménagement d'une zone dédiée aux pique-niqueurs dès l'entrée de la route forestière afin de leur interdire les espaces les plus sensibles. Succès manifeste, cette stratégie a eu pour conséquence une concentration automobile au bord du somin Volcan en déconnexion totale des labels de cœur de parc national et de bien du patrimoine mondial affichés pour le massif de la Fournaise.

Parallèlement à la valorisation du patrimoine naturel de l'île, la période contemporaine a été marquée par une interpellation identitaire. Cette interrogation a eu comme terreau indirect une prise de conscience liée au vécu quasi quotidien de l'uniformisation rapide des paysages urbanisés du littoral, dont l'extension en direction du sommet des planèzes extérieures a effacé, dans un même mouvement, les principaux repères spatiaux de la culture créole dans les Bas de l'île. Retrouver ces marqueurs, pour une partie de la population, nécessite alors de se tourner vers les Hauts et le cœur de cirques considérés comme les derniers refuges de la créolité (Robert, 2006: 8 et 10).

Ce réflexe identitaire constitue une chambre d'écho particulièrement favorable pour les récits du tan lontan dont les résonances sensibilisent une part grandissante de la société réunionnaise à la recherche des signes de ses identités. Les histoires mises en dramaturgie des premiers arrivants et celles des suivants, engagés volontaires ou non, ainsi que les relectures des souvenirs du «temps de la colonie», toutes participent à une compilation de récits devenue indispensable pour conforter les enjeux patrimoniaux de l'île. Depuis plusieurs années, le vif intérêt dont jouissent les suppléments insérés chaque dimanche dans les quotidiens de l'île, en révélant à travers des fonds de photographies anciennes, les divers aspects oubliés de la vie lontan, témoigne du désir de la population de se réapproprier un passé, pas si éloigné, mais trop vite enseveli sous les transformations profondes que l'île a connues depuis les années 1960-1970.

18 Le site Internet du parc national de La Réunion consacre une page aux dégâts causés à la végétation par les grands incendies. Sur l'épisode qui a eu lieu au volcan en 2010, voir: http://www.reunionparcnational.fr/Fin-de-l-incendie-au-Volcan.html.

19 Parallèlement à une augmentation de la fréquentation des Hauts de l'île par le développement des loisirs de plein air (Bouchet et Gay, 1998), les premiers résultats encore inédits des travaux menés par notre collègue Télesphore Brou (équipe Espace-Dev) semblent accréditer l'hypothèse d'une corrélation entre le nombre des débuts d'incendie et les changements climatiques sur les parties hautes des massifs volcaniques de La Réunion, en particulier pour la planèze sous le vent du Maïdo. 
Ce contexte, associé à une acceptation généralisée de la posture intellectuelle prônant une conciliation entre les parts immatérielle et naturelle du patrimoine réunionnais, ouvre désormais une large perspective conceptuelle au réenchantement du somin Volcan.

\section{Interprétation et valorisation écotouristique du somin Volcan}

Inscrite dans les partis pris de la rénovation de la Maison du Volcan (ouverte en 1992), la volonté de mieux prendre en compte la mémoire des découvreurs de la Fournaise accorde une place centrale aux voyages des «hommes aux semelles volcan». Dans ce nouveau cadre, l'exhumation du somin Volcan présente l'intérêt de répondre conjointement à la politique de desserrement des flux de visiteurs au bord de la Fournaise et à celle de valoriser les liens historiques entre les hommes et le volcan, tout en fournissant un axe fort de mise en scène pour le Schéma d'interprétation et de valorisation écotouristique du massif volcanique (Sicre, 2007). Le schéma épousant la posture vertueuse du développement durable exigée par tous les voyagistes, son originalité réside surtout dans le terme «interprétation». Formalisé par Tilden (1957), ce concept procède de l'enquête de cet auteur auprès des responsables des parcs nationaux américains à propos du ressenti des visiteurs interrogés après leur découverte du parc. Si les personnes ont été conquises par la visite et par le caractère remarquable des paysages observés, les explications scientifiques fournies au gré du parcours emprunté ont été diversement appréciées. Plus que le contenu, peu contestable, c'est surtout la forme univoque et, par moments pontifiante, du langage utilisé qui ne les a pas réellement convaincus. Ce constat conduit Tilden à énoncer six principes fondateurs supposés renouveler l'expérience de la visite des grands parcs nationaux (figure 4).

Inspiré par la réflexion de Tilden ${ }^{20}$, le Schéma d’interprétation et de valorisation écotouristique (SIVE) de la Fournaise intègre les fondamentaux de l'interprétation à partir d'une lecture centrée sur les éléments de caractère du Piton de la Fournaise et sur les enjeux de sa patrimonialisation. Conçu par Michel Sicre, le scénario propose un voyage dans le temps et dans l'espace dont le vecteur circulatoire est calqué, dans ses grandes lignes, sur les traces de l'ancien somin Volcan. L'itinéraire, comme objet spatial linéaire, est donc bien l'acteur central du schéma sur lequel s'articule l'ensemble des sites d'interprétation: le pays des hommes, la lande mystérieuse et le monde des origines (figure 5). Dans chacune des trois séquences paysagères distinguées (le rural, le végétal et le minéral), le recours aux récits du tan lontan à travers les épopées des premiers voyageurs à la Fournaise est une constante permettant de valoriser le dispositif d'interprétation. Celui-ci est conceptualisé en quatre étapes scénarisées sur le message «le volcan, cœur du monde et cœur des hommes». En fondant sa construction sur les grands récits volcanologiques de la Fournaise, le voyage entraîne le visiteur à cheminer depuis sa naissance (Plaine des Cafres) jusqu’à ses manifestations contemporaines (belvédère de Bellecombe). L’occurrence réelle d'un épisode volcanique qu'on peut observer en direct ajoute un attrait supplémentaire à un scénario déjà très riche.

20 Après un certain effacement, l'application du concept d'interprétation de Tilden semble trouver un second souffle avec l'essor de la valorisation des patrimoines (Drouin, 2007). En inscrivant au cœur de sa charte la valorisation des paysages par l'interprétation, le parc national de La Réunion illustre cette évolution, voir: http://www.reunion-parcnational.fr/Enquete-publique/Essentiel-A4-32pages.pdf, p. 21. 
Figure 4 Le concept d'interprétation de Freeman Tilden

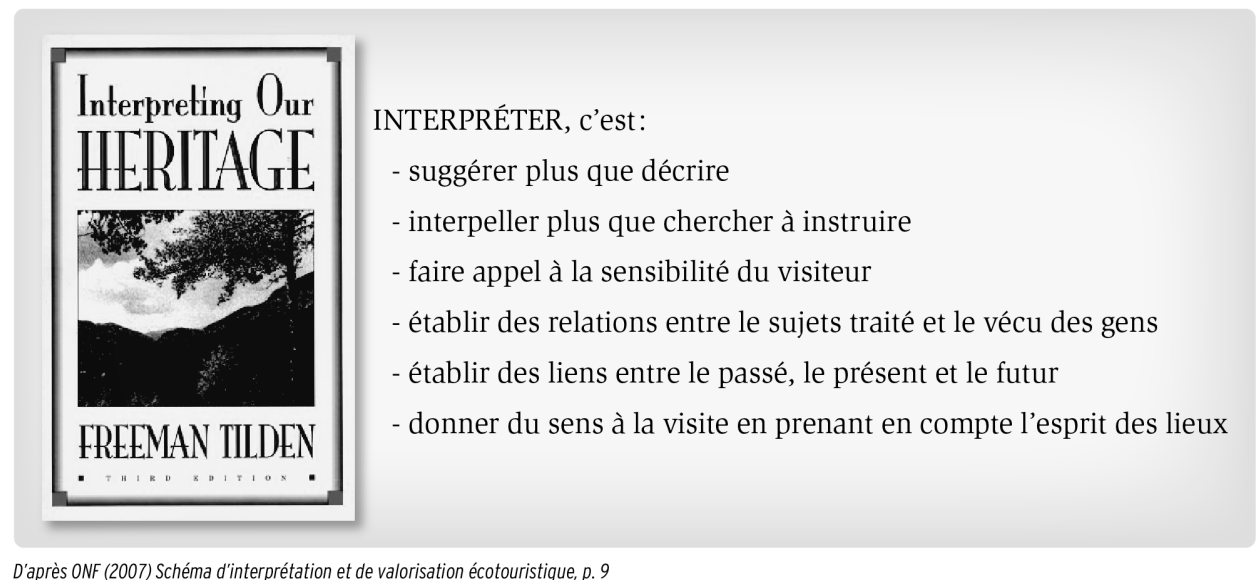

Les trois effondrements successifs scandent le rythme et les étapes de la rencontre avec le volcan (figure 5) et, à chacune des haltes, des éléments de mise en scène accompagnent la découverte spectaculaire du paysage qui sert de clé de lecture et de mesure entre le temps de la terre et le temps des hommes. L'itinéraire qui relie ces escales paysagères n'est plus un simple élément de passage, mais il devient en lui-même un objet de médiation permettant de renouer momentanément avec la dimension initiatique attachée à la conquête du massif de la Fournaise. Cette mise en scène du somin Volcan soutient le principe d'un aménagement minimaliste et totalement intégré au site. Il ne s'agit pas d'investir dans de gros travaux, ni de multiplier les éléments de signalétique. Certains points de vue seront réaménagés et adaptés aux personnes à mobilité réduite (Pas de Bellecombe) ou, comme celui du Nez de Bœuf, démultipliés pour éviter les phénomènes de concentration et supprimer des stationnements délicats et trop visibles. La volonté est de faire du somin Volcan, non plus une simple trajectoire entre destinations (Bourg-Murat - enclos Fouqué), mais un véritable espace de destination. La construction de nouveaux récits (SIVE), le choix d'une protection environnementale labellisée (parc national, UNESCO) et l'instauration des politiques de patrimonialisation (Région Réunion et conseil régional) sont, sans doute, de nature à remodeler l'attractivité de la route du volcan, mais pour quels usages et pour quels publics? 


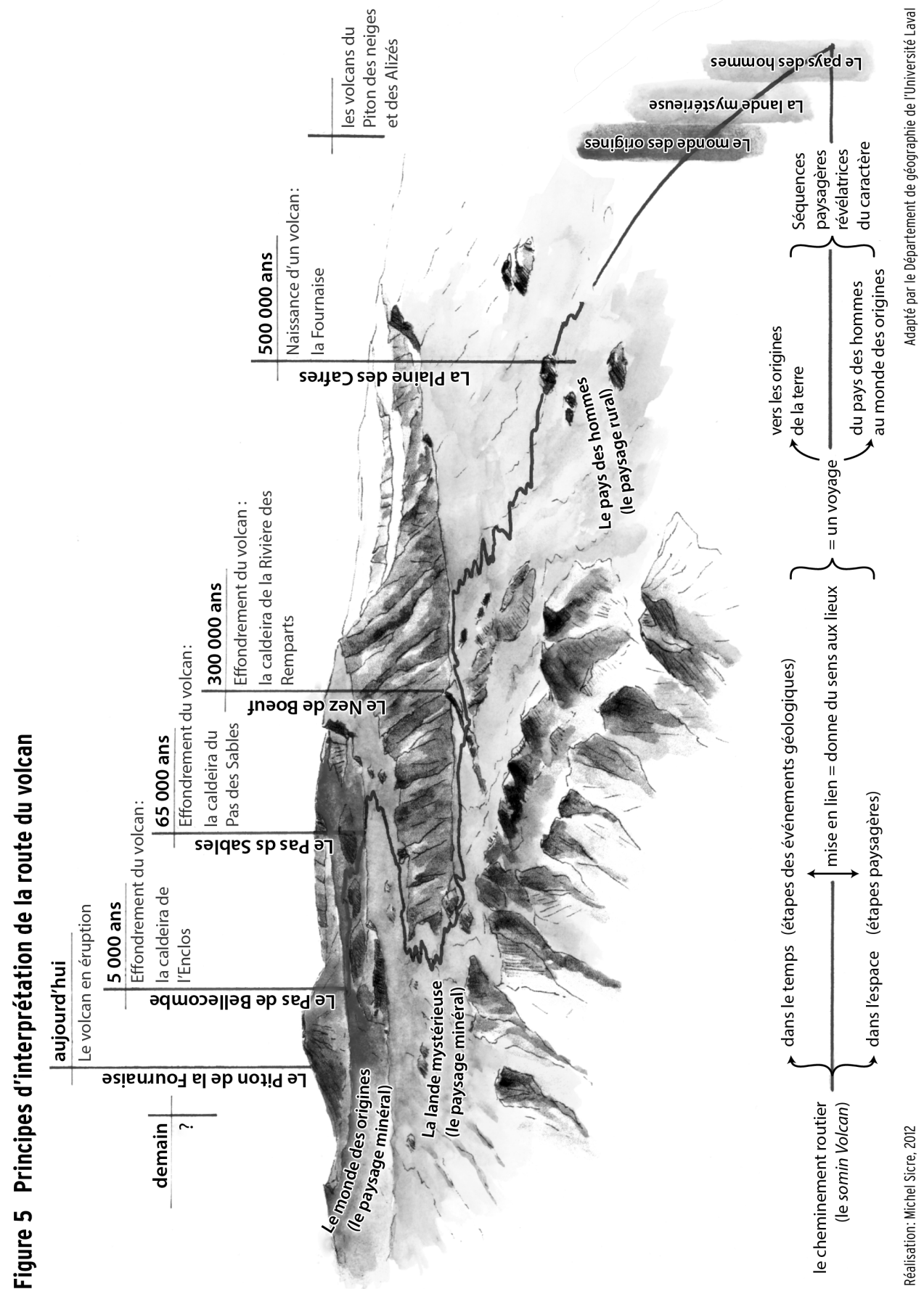




\section{La Route du Volcan, une authenticité encadrée}

Si le SIVE de la Fournaise tente de renouer avec l'esprit du somin Volcan, nous devons constater que la réalité dessinée est tout autre. Déjà, dans l'appellation de l'itinéraire, nous sommes passés du somin Volcan, à la «route forestière du Volcan», pour aboutir à la «Route du Volcan», expression en cours d'officialisation auprès des responsables de la région pour désigner le projet de la mise en scène de la route forestière, l'une des neuf unités d'interprétation du SIVE de la Fournaise. Il faut préciser que le parc national et la Région Réunion procèdent actuellement à l'aménagement d'une autre unité sur le même territoire d'interprétation (le massif du volcan) : la Route des Laves correspondant au tronçon routier qui traverse le Grand Brûlé entre les villages de Piton Sainte-Rose et du Tremblet. Le choix du terme «Route», dans les deux cas, n’est pas insignifiant. Tout en reconnaissant la nécessité de limiter l'augmentation des flux de véhicules sur les hauts du volcan et en souscrivant au principe d'ouverture ou de réaménagement des chemins pédestres adjacents à l'itinéraire principal, le financement régional engagé ne prend en compte que la route et ses «arrêts-minute». Pour les acteurs du développement touristique de la région, l'automobile reste de façon incontestable le vecteur prioritaire pour la découverte de la Fournaise. Compte tenu de la situation actuelle relative aux possibilités et aux moyens de transport sur l'île et au volcan, ce parti pris pragmatique est cohérent, même s'il peut être en contradiction avec les recommandations de la charte du parc national de La Réunion et les recommandations induites par le label «patrimoine mondial». Sans développer les débats engagés sur ce choix, ils révèlent au moins une réalité contemporaine: la grande diversification des pratiques touristiques et de loisir au sein des sociétés contemporaines. Le constat n'est pas nouveau et la littérature sur ce sujet est abondante (Équipe MIT, 2011 ; Bourdeau et al., 2013). Ainsi, il n'existe plus une seule manière «d'aller au volcan» comme cela était sans doute le cas au cours de la première période du somin Volcan, mais différentes façons de le visiter, chacune adaptée aux représentations et aux motivations des divers groupes de visiteurs qui entreprennent l'excursion. À chacun sa route ou son somin.

En effet, dans une approche qui tient compte des nouveaux objectifs guidant les curieux à la Fournaise et en relation avec la thématique somin Volcan/Route du Volcan, nous pouvons distinguer schématiquement trois groupes de visiteurs faisant un usage différent de l'itinéraire.

Le premier correspond aux aficionados, amateurs passionnés et puristes du Piton de la Fournaise. La mixité de sa composition sociale réunit des résidants de longue durée, des visiteurs temporaires et des habitants de l'île dont l'histoire personnelle se rattache au volcan. Dans ces derniers, figurent les enfants des curieux initiaux et de leurs guides (familles Bénard, Gérard, Cornu, André, Picard), nourris des récits du voyage de leurs parents à la Fournaise. Ils ont développé une véritable culture du volcan et pratiquent généralement le hors-piste vis-à-vis de la route forestière. Aujourd'hui, ces marcheurs impénitents sont sans doute, parmi l'ensemble des visiteurs, les plus attachés à la réhabilitation du somin Volcan dans sa version pédestre originelle. En l'empruntant régulièrement, ils tendent à effacer la «vacance» de certaines de ses parties réduites à l'état de «friche» (Bachimon, 2013) depuis l'ouverture de la route forestière. Leur compilation acharnée des images anciennes et des zistwar du volcan en font de fins connaisseurs de son histoire et des zélateurs convaincants d'une patrimonialisation «ouverte» du massif de la Fournaise. Si l'effectif de ce groupe informel est assez 
restreint, moins de 200 personnes en tout, son influence n'est pas négligeable dans la mesure où ses membres, qui se renouvellent au gré des circonstances, sont souvent présents dans des commissions, des services ou des associations intervenant sur les grands projets d'aménagement de l'île. Certains sont même des journalistes reconnus et publient régulièrement des chroniques défendant une pratique libertaire mais respectueuse des parcours du volcan.

Le second ensemble est constitué en majorité de touristes de l'extérieur de l'île. Souvent accompagnés par des habitants ou des résidants avec lesquelles ils entretiennent des liens affinitaires, ils sont les plus nombreux à visiter chaque année le volcan. Selon les estimations les plus récentes, produites à l'interne par le parc national (2011), ils seraient presque 350000 au bord de l'Enclos, et environ 60000 à entreprendre l'ascension de la Fournaise. La mise en scène de la Route du Volcan s'adresse plus particulièrement à ce groupe dont on espère limiter la concentration au Pas de Bellecombe et surtout allonger la durée de sa visite. Si la route forestière et ses trois haltes traditionnelles (Nez de Bœuf, Commerson, Plaine des Sables) répondent à l'essentiel des attentes de ce groupe (accès motorisé rapide et aisé à la Fournaise, explication générale de l'histoire de son volcanisme lors des «arrêts-minutes»), la présentation des récits, des points de vue paysagers et des offres de découverte introduite par le SIVE doit l'inciter à passer plus de temps sur le massif et à renouveler la fréquence de ses passages. La Maison du Volcan, dont la réouverture était programmée pour le mois d'avril 2014, s'avère un dispositif clé de la Route du Volcan en introduisant, à travers plusieurs plaquettes disponibles pour les visiteurs, les sommaires du récit de sa mise en scène et les descriptions des «itinérances» associées.

Le dernier groupe de visiteurs rassemble les adeptes du pique-nique dominical ou du week-end qui se sont approprié l'espace du volcan le long de la route forestière selon la disponibilité des kiosques mis à disposition par l'ONF. Il est composé principalement des habitants de l'île issus des hautes plaines et du littoral, dont la visite à la Fournaise n'implique pas forcément d'accéder à l'Enclos ou de se rendre au sommet du Piton, ni d'ailleurs de chercher à comprendre les détails morphologiques et volcanologiques de l'histoire du massif. Ces usagers de la route forestière transposent, à l'altitude du volcan, les rites de sociabilité observables plus facilement sur les plages, consistant à réunir la famille et les amis autour d'un «cari volaille» ou d'un «rougail saucisses» afin de cultiver les solidarités en partageant un moment enjoué, ponctué par les tonitruantes parties de dominos. L'entreprise est rendue très visible par l'amoncellement des marmites et par la disposition de bâches en plastique bleues tendues autour des abris pour se protéger de la pluie et du vent. Utilisant du bois mort collecté à proximité du bivouac ou du charbon de bois transporté depuis les Bas, un foyer complète l'équipement de la compagnie. L'importance du nombre de campeurs et pique-niqueurs de la route forestière est mal évaluée. L'estimation rudimentaire qui est retenue, 100000 personnes (2011), est déduite du nombre de visiteurs à l'entrée de la route du volcan et de celui des curieux arrivés au bord de l'Enclos.

Institutionnalisée par le parc national et promue par l'UNESCO comme vecteur de découverte des paysages remarquables inscrits au patrimoine mondial, la Route du Volcan apparaît comme une construction spatiale dont l'authenticité réinventée reste très encadrée. Si cet environnement semble convenir aux visiteurs de l'extérieur, les fervents du somin Volcan et les familiers de la route forestière sont très dubitatifs face 
à cette réalisation. C'est particulièrement le cas pour les derniers, souvent pointés (injustement?) par les agents du parc comme le groupe présentant un risque important de dégradation pour les milieux du volcan. Sans vouloir les exclure, la solution envisagée est le démontage progressif des kiosques dans la partie haute du volcan pour rassembler les pique-niqueurs dans des zones aménagées en conséquence à l'entrée de la route forestière, à la hauteur du Piton Sec, l'affluence concentrée de ces visiteurs et de l'automobile étant considérée comme un moindre mal dans une aire où la végétation endémique est peu présente et où les risques de début d’incendie restent limités. De nature à générer un clivage entre somin Volcan et Route du Volcan, ces décisions ne seront pas sans conséquence lorsque l'on connaît l'attachement des habitants de l'île à ces pratiques récréatives ${ }^{21}$.

\section{Discussion}

Quelle utilité ou quel intérêt pouvons-nous retenir de cette brève incursion sur le somin Volcan de La Réunion en rapport avec la thématique de ce dossier Routes touristiques : lire le passé, lier l'avenir?

Sur un plan purement factuel, l'exemple du somin Volcan s'inscrit sans réelle surprise dans la trajectoire généalogique des places touristiques. Que ce soit dans son invention ou dans son histoire (banalisation, patrimonialisation), il ne présente pas une profonde singularité par rapport aux parcours des autres hauts lieux touristiques. En particulier, les dynamismes évolutifs identifiés s'accordent sans difficulté aux interprétations renouvelées des facteurs d'évolution d'un lieu touristique (Équipe MIT, 2011). Nous pouvons rappeler la définition du haut lieu proposé par Debarbieux applicable au champ du tourisme: «Le haut lieu est [...] à la fois une localisation géographique particulière, vécue comme étant singulière en raison de sa forte charge symbolique, et un lieu qui rend possible l'expression d'une adhésion individuelle à une idéologie collectivement partagée» (Debarbieux, 2003: 448-449). En accord avec cette assertion, la figure 6 propose l'esquisse d'un modèle d'évolution du haut lieu touristique linéarisé. Elle synthétise les processus de valorisation de l'itinéraire en précisant la transformation de sa géographie ainsi que celle des postures paysagères attachées à l'observation du volcan et applicables plus largement au domaine de la montagne. Les historiens et les géographes ont bien décrit les processus de l'invention de la montagne et de la transfiguration du paysage (Joutard, 1986; Debarbieux, 1988 et 2001; Briffaud, 1994) qui se retrouvent assez fidèlement pour le Piton de la Fournaise. Dans ce mouvement, le somin Volcan est un vecteur essentiel dont la spatialité élargie au cours de la dernière période ouvre de nouvelles pistes de découverte et (re)produit de nouveaux récits paysagers livrant l'intégralité du massif volcanique à la curiosité des touristes. La première période (1750-1965) est le temps des «découvreurs», voire des «pratiquants» de la Fournaise eu égard à leur dévotion du volcan, qui construisent la spatialité de l'itinéraire. Celle-ci est traduite par le somin Volcan qui s'impose comme l'axe d'attraction de la Fournaise. Structuré sur deux haltes paysagères, l'itinéraire aboutit au bord de l'enclos Fouqué qui marque généralement la fin du voyage. La partie ouest du Piton de la Fournaise

21 Les articles du Journal de l'Île (13 janvier 2014) sur la contestation du démantèlement du kiosque du Petit Bois d’Ozoux et les réactions des lecteurs illustrent parfaitement ce risque de clivage. Voir en particulier: Colère autour du démantèlement d'une aire de pique-nique chemin volcan et les 43 commentaires associés à l'article. Il est intéressant de noter la mobilisation de l'expression «chemin volcan» (http://www.clicanoo.re/401629-colere-autour-du-demantelement-d-une-aire-depique-nique-chemin-volcan.html). 
observée depuis ce point de vue va progressivement incarner la représentation normative du volcan. Si cette première séquence construit une spatialité à l'échelle du local, la suivante (1965-fin des années 1990) investit celle du national par un élargissement géographique des flux de visiteurs. La spatialité produite est plus complexe par la diversité des pratiques que l'ouverture de la route forestière entraîne. Du phénomène volcanique apprivoisé par la connivence paysagère développée chez les visiteurs initiaux, la seconde période valorise le volcan comme attraction spectaculaire dont la consommation massive implique l'entrée en scène de l'aménageur. Ce dernier instruit, à l'usage des nouveaux visiteurs, une lecture pédagogique du paysage de la Fournaise dont l'itinéraire codifié amplifie la focalisation sur le parcours de la route forestière. La période actuelle est encore plus riche au niveau des échelles en mêlant les deux premières, attachées aux temps précédents, à celle de l'international, rendue effective par la labellisation du volcan comme patrimoine mondial de l'UNESCO. Conforme aux politiques de préservation et de valorisation exigées pour les biens inscrits sous ce label, cette stratégie de patrimonialisation vise à atténuer la pression des flux de curieux sur les bords de l'enclos Fouqué et au sommet du volcan. Le projet de la Route du Volcan doit permettre de réaliser cet objectif en élargissant la spatialité antérieure par l'ouverture et la réhabilitation d'un réseau d'itinéraires greffé sur l'axe principal: sentier du sommet du Morne Langevin, chemin du Piton de l'Eau, de Foc Foc... Le choix du parti pris d'un recours au passé des paysages et à leur projection dans le futur est aussi un élément essentiel pour permettre aux visiteurs de mieux percevoir les enjeux d'inscrire, sur le long terme, une politique de développement touristique durable, non seulement pour le massif de la Fournaise mais pour l'ensemble du territoire réunionnais.

L'originalité présentée par le somin Volcan pour cette réflexion se situe, de notre point de vue, dans la méthode d'interprétation et de valorisation écotouristique proposée par le SIVE de la Fournaise. Adaptable, donc a priori transférable, cet outil constitue une réponse plausible aux préoccupations formulées par les responsables de l'UNESCO qui s'interrogent sur la mobilisation d'un tourisme responsable pour la valorisation des sites inscrits au patrimoine mondial (colloque de Libreville en juin 2012). Il faut cependant souligner deux écueils importants attachés au plan d’interprétation. Le premier est la difficulté de sa mise en œuvre par une gouvernance feuilletée très lourde nécessitant la coordination d'un trop grand nombre d'acteurs, institutionnels, privés et associatifs. Le second renvoie au danger d'introduire un clivage profond entre les usagers. Les habitants des espaces d'application des schémas d'interprétation et de valorisation écotouristique se sentent souvent exclus par ce nouvel aménagement qui, en réencadrant leurs pratiques, les contraint à restreindre leurs spatialités coutumières, d'où la naissance d'un sentiment de dépossession.

Enfin, sur un plan plus théorique, l'exemple du somin Volcan permet de réfléchir sur la spécificité du haut lieu touristique comme espace linéarisé. Mais cette spécificité ne réside-t-elle pas plus dans la nature de l'objet touristique que dans son expression spatiale? Dans ce cas, à quoi bon s'interroger sur la ligne (routes, circuit, chemin...) comme objet touristique spécifique? Ce serait sans doute oublier un peu vite que les hauts lieux linéarisés, qu'ils soient route du sel ou de la soie, ligne céleste de Mermoz ou océanique des Polynésiens, chemin menant à Saint-Jacques-de-Compostelle ou à Machu Picchu, sont tous profondément attachés à un imaginaire collectif qui valorise la figure du nomade. Et c'est sans doute, dans son lien à la posture idéelle du nomadisme que se singularise, aujourd'hui, la ligne parmi les hauts lieux touristiques avec sa vitalité animée par une mobilité circulatoire, imaginée comme une histoire sans fin et pratiquée comme un voyage «au long cours» dont la destination se décale sans cesse vers l'ailleurs. 


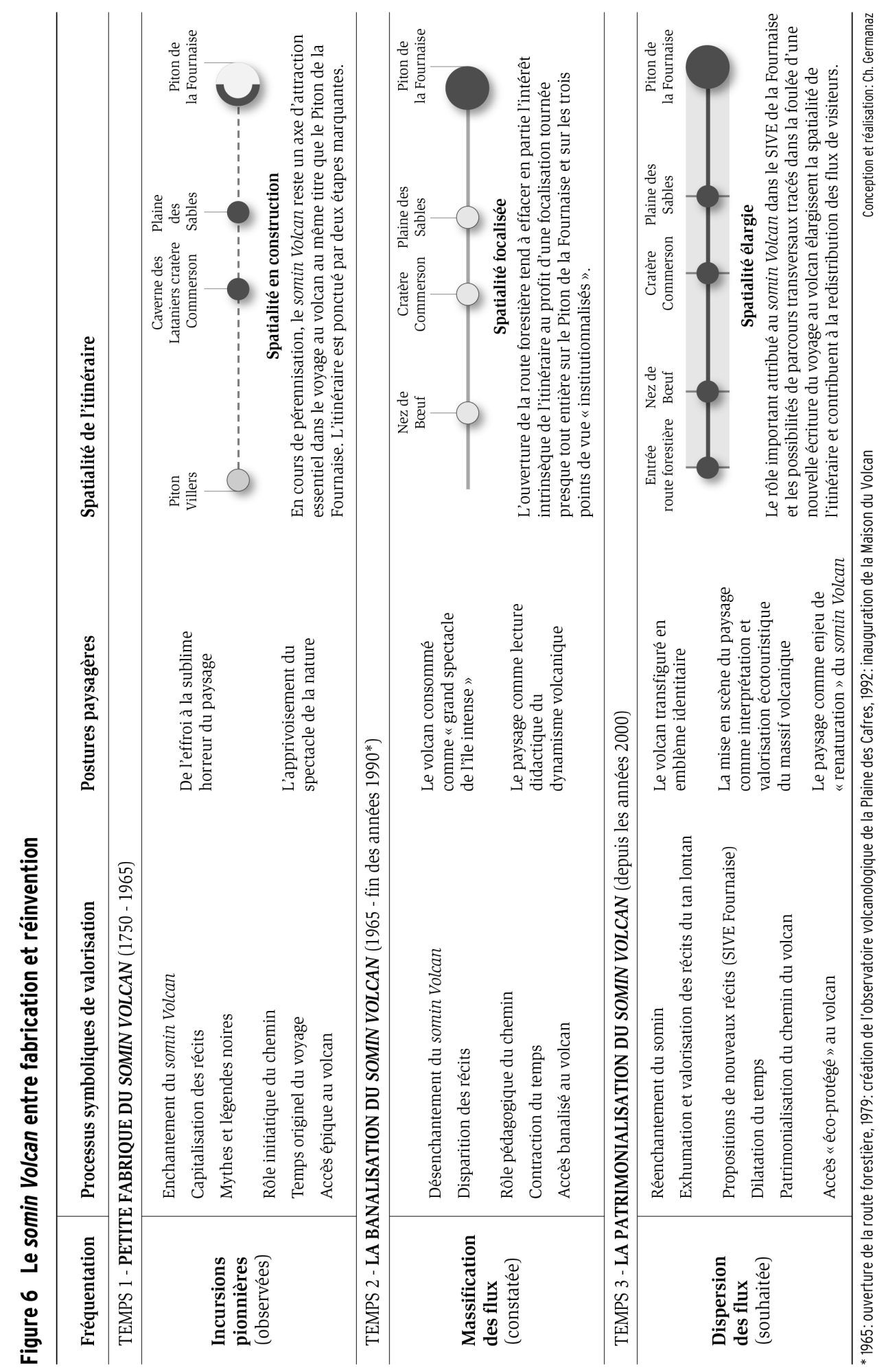




\section{Bibliographie}

AVICE, Bertrand (2001) L'écotourisme sur la façade orientale du massif du Piton de la Fournaise, île de La Réunion-Océan Indien: définition, enjeu et stratégie d'aménagement dans le cadre d'un développement durable. Saint-Denis (La Réunion), Université de La Réunion, Département de géographie, thèse de doctorat.

BACHIMON, Philippe (2013) Vacance des lieux. Paris, Belin.

BORY de SAINT-VINCENT, Jean-Baptiste (1804) Voyage dans les quatre principales illes des mers d'Afrique, fait par ordre du gouvernement, pendant les années 9 et 10 de la République (1801-1802). Paris, Buisson.

BOUCHET, Patrick et GAY, Jean-Christophe (1998) Les Hauts de la Réunion conquis par les loisirs, Mappemonde, vol. 51, nº3, p. 31-37.

BOURDEAU, Philippe, PERRIN-BENSAHEL, Liliane et FRANÇOIS, Hugues (dir.) (2013) Fin (?) et confins du tourisme. Interroger le statut et les pratiques de la récréation contemporaine. Paris, L'Harmattan.

BRIFFAUD, Serge (1994) Naissance d'un paysage. La montagne pyrénéenne à la croisée des regards, XVI ${ }^{e}$-XIX ${ }^{e}$ siècles. Tarbes / Toulouse, Archives de Hautes/Pyrénées-Université de Toulouse.

DEFOS du RAU, Jean (1960) La Réunion, étude de géographie humaine. Bordeaux, Institut de géographie, thèse de doctorat.

D'HEGUERTY (1755) Observations sur le volcan de l'Isle de Bourbon. Mémoires de la Société royale des sciences et belles lettres de Nancy, tome troisième, p. 218-230.

DEBARBIEUX, Bernard (1988) Territoires de haute montagne: recherches sur le processus de territorialisation et d'appropriation sociale de l'espace de haute montagne dans les Alpes du Nord. Grenoble, Université de Grenoble 1, Institut de géographie, thèse de doctorat.
DEBARBIEUX, Bernard (2001) Différenciation et désignation géographique des objets alpins: six manières de faire. Revue de géographie alpine, vol. 89, nº4, p. 43-65.

DEBARBIEUX, Bernard (2003) Haut lieu. Dans Jacques Lévy et Michel Lussault (dir.) Dictionnaire de la géographie et de l'espace des sociétés. Paris, Belin, p. 448-449.

DROUIN, Martin (2007) Un vieux débat toujours ranimé autour de l'interprétation du patrimoine. Téoros, vol. 26, $\mathrm{n}^{\circ} 3$, p. 72-75. [En ligne]. http://teoros.revues. org/1042

GAMALEYA, Boris (1983) Le Volcan à l'envers ou madame Desbassyns le diable et le bondieu. Saint-Leu (La Réunion), Presses de développement.

GERMANAZ, Christian (2004) Du dessous du volcan au bord des cratères: l'invention du paysage volcanique du Piton de La Fournaise (La Réunion). Dans Dominique Bertrand (dir.) L'invention du paysage volcanique. Clermont-Ferrand, Presses universitaires Blaise Pascal, p. 259-283.

GERMANAZ, Christian (2005a) Du pont des navires au bord des cratères: regards croisés sur le Piton de la Fournaise (16531964). Itinéraires iconographiques et essai d'iconologie du volcan actif de La Réunion. Paris, Université Paris-Sorbonne, thèse de doctorat.

GERMANAZ, Christian (2005b) Iconographie, imaginaire et géographie: quelques étapes dans l'évolution des représentations sur le Piton de la Fournaise (volcan de la Réunion). Dans Françoise Sylvos et Marie-France Bosquet (dir.) Magma Mater. L'imaginaire du volcan dans l'Océan Indien, Paris-Saint-Denis de la Réunion, SEDES-Université de La Réunion, p. 72-88. 
GERMANAZ, Christian (2009) L'image du Piton de la Fournaise dans les stratégies touristiques de La Réunion. Dans Dominique Bertrand, Daniel Lieutort et Jean-Claude Thouret (dir.) Villes et volcans. Clermont-Ferrand, Presses Universitaires Blaise Pascal, p. 45-70.

GERMANAZ, Christian (2013) Le Piton de La Fournaise: ce qu'en disent les images. Iconologie du paysage volcanique de La Réunion. Dans Dominique Poulot (dir.) Paysage et Iconographie (135e Congrès national des sociétés historiques et scientifiques, Neuchâtel, 2010). Paris, CTHS, p. 57-70.

HERMANN, Jules (1890) Excursion au volcan et observations sur son activité. Bulletin de l'Académie de l'île de La Réunion, volume de l'année 1890, p. 77-83.

HÉRY, Louis (1883) Fables créoles et explorations dans l'intérieur de l'île Bourbon. (Esquisses africaines). Paris, nouvelle éd., J. Rigal.

HUBERT, Jean-Joseph (1799) Lettre manuscrite du 8 novembre 1799, adressée au Comte Favéta. (Le document est repris dans Émile Trouette (1882) Papiers de Joseph Hubert, Saint-Denis de La Réunion, Lahuppe).

JACQUARD, Fabrice (2009) Étude de la vulnérabilité humaine via la perception des risques sur les sentiers de randonnée pédestre de l'île de La Réunion - Étude de cas: Ravine Saint-Gilles, Formica Léo, Chapelle Rosemont, Cratère Kapor, Cratère Rivals, Tour des Cratères. Université de La Réunion, Département de géographie, mémoire de master.

JACOB DE CORDEMOY, Eugène (1867) Itinéraire d'un voyage au volcan en 1862 . Dans Antoine Roussin (dir.) Album de la Réunion: recueil de dessins et de textes historiques et descriptifs. Saint Denis, Lahuppe, tome IV, p. 108-118.

JOUTARD, Philippe (1986) L'invention du Mont Blanc. Paris, Gallimard.

KRAFFT, Maurice et KRAFFT, Katia (1981) Dans l'antre du diable. Volcans d'Afrique, Canaries et Réunion. Paris, Presses de la Cité.
LUSSAULT, Michel (2003) Spatialité. Dans Jacques Lévy et Michel Lussault (dir.) Dictionnaire de la géographie et de l'espace des sociétés. Paris, Belin, p. 866-868.

MAS, Magali (2012) Analyse comparative des représentations du risque volcanique en milieu insulaire-Guadeloupe, Martinique et Réunion. Université Montpellier III, thèse de doctorat de géographie.

MERWART, Émile (1929) Pour accéder au volcan. Saint-Denis (Réunion). La Victoire Sociale (journal de l'île de La Réunion), édition du samedi 5 janvier 1929, n 5053 , p. 1.

Équipe MIT (2011) Tourismes 3. La révolution durable. Paris, Belin.

MORIN, Julie (2012) Gestion institutionnelle et réponses des populations face aux crises volcaniques: études de cas à La Réunion et en Grande Comore. Saint-Denis de La Réunion, Université de La Réunion, Départements de géographie et des sciences de la terre, thèse de doctorat.

OFFICE NATIONAL DES FORÊTS (ONF) (2007) Schéma d'interprétation et de valorisation écotouristique. Saint-Denis, ONF-Réunion, Cahier 1, p. 9.

OZOUX, Louis (1938) Une excursion au volcan. Dans Louis Ozoux (dir.) Conférences et études. Port Louis (Île Maurice), The General Printing \& Stationery. CY-LTD, p. 181-212.

PAYET, Gilda Marie Sylvine (2005) Tourisme et environnement naturel, le cas de la route forestière du volcan. Saint-Denis de La Réunion, Université de La Réunion, Département de géographie, mémoire de maîtrise.

PETIT-RADEL, Philippe (1801) De amoribus Pancharitis et Zoroœ, poema eroticodidacticon. Parisiis, Didot junior.

ROBERT, René (2003) Les Pluies médianes. Dans CREGUR et INSEE-Réunion, Atlas de La Réunion. Saint-Denis, Université de La Réunion, p. 26-27. 
ROBERT, René (2006) Les destinations du Parc Naturel. Dans René Robert (dir.) Dossier d'enquête publique, Cahier 5. État des lieux et du patrimoine. Saint-Denis (Réunion), Mission du Parc National, p. 60-61.

ROBERT, René (2009) Île de La Réunion, un patrimoine naturel d'exceptions. Une présentation simplifiée des travaux réalisés pour la candidature de La Réunion au Patrimoine Mondial de l'Unesco. SaintDenis de La Réunion, Parc national de La Réunion.

SICRE, Michel (2007) Schéma d'interprétation et de valorisation écotouristique: Massif de La Fournaise, le volcan, cœur du monde, cœur des hommes. Saint-Denis, Office national des forêts, Direction régionale de La Réunion.

TILDEN, Freeman (1957) Interpreting our heritage: principles and practices for visitor services in parks, museums, and historic places. Chapel Hill, University of North Carolina Press. 